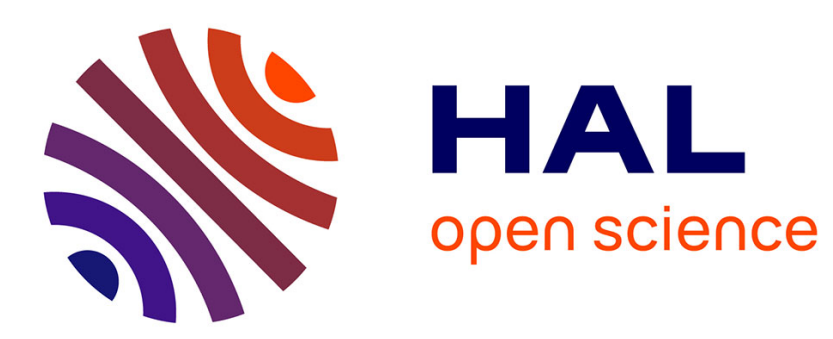

\title{
Study of permeability by periodic and self-consistent homogenisation
}

Claude Boutin

\section{To cite this version:}

Claude Boutin. Study of permeability by periodic and self-consistent homogenisation. European Journal of Mechanics - A/Solids, 2000, 19 (4), pp.603-632. hal-00943756

\section{HAL Id: hal-00943756 https://hal.science/hal-00943756}

Submitted on 8 Feb 2014

HAL is a multi-disciplinary open access archive for the deposit and dissemination of scientific research documents, whether they are published or not. The documents may come from teaching and research institutions in France or abroad, or from public or private research centers.
L'archive ouverte pluridisciplinaire HAL, est destinée au dépôt et à la diffusion de documents scientifiques de niveau recherche, publiés ou non, émanant des établissements d'enseignement et de recherche français ou étrangers, des laboratoires publics ou privés. 


\title{
Study of permeability by periodic and self-consistent homogenisation
}

\author{
Claude Boutin ${ }^{1}$ \\ Ecole Nationale des Travaux Publics de l'Etat, Laboratoire Géomatériaux, DGCB, URA CNRS N 1652, Rue Maurice Audin, \\ 69518 Vaulx-en-Velin Cedex, France
}

\begin{abstract}
This paper presents a study into permeability of porous media in which both homogenisation of periodic media (HPM) and self-consistent method (SCM) are used. By taking advantage of the physical principles identified with HPM, the application of SCM led to the determination of two permeability assessments. It is shown that these conjectured values correspond in fact to the exact bounds of permeability of various classes of porous media with a clear identification of their microstructures (grain and fluid size distribution). Applications are presented for granular media with spherical grains, and for fibrous media with parallel cylindrical fibres.

homogenisation of periodic structure / self consistent method/permeability / Navier-Stokes equation / granular media / fibrous media
\end{abstract}

\section{Nomenclature}

$E_{\mathrm{A}}, E_{\mathrm{B}}, E_{I} \quad$ Dissipated powers, in medium A, medium B, inclusion $I$.

$E_{\mathrm{c}}\left(\mathbf{v}_{\mathrm{p}}, \mathbf{v}_{\mathrm{v}}\right) \quad$ Dissipated power between any kinematically and statically continuous fields.

$H_{\mathrm{p}} \quad$ Higher bound of the intrinsic permeability of medium PM based on statically continuous fields.

$H_{\mathrm{v}} \quad$ Lower bound of the intrinsic permeability of medium PM based on kinematically continuous fields.

$I(R, \rho) \quad$ Spherical inclusion characterised by $R$ and $\rho$.

$K_{\mathrm{p}}=R^{2} \Psi_{\mathrm{p}}(\rho) \quad$ Intrinsic permeability of the Darcy medium statically consistent with the spherical inclusion $I(R, \rho)$.

$K^{\mathrm{p}} \quad$ Actual intrinsic permeability of a Darcy medium $\mathrm{PM}_{\mathrm{p}}$.

$K_{\mathrm{v}}=R^{2} \Psi_{\mathrm{v}}(\rho) \quad$ Intrinsic permeability of the Darcy medium kinematically consistent with the spherical inclusion $I(R, \rho)$.

$K^{\mathrm{v}} \quad$ Actual intrinsic permeability of a Darcy medium $\mathrm{PM}_{\mathrm{v}}$.

$P^{\mathrm{e}} \quad$ Pressure in the Darcy medium such that $\operatorname{grad}\left(P^{\mathrm{e}}\right)=\mathrm{e}_{z}$.

$\mathrm{PM}_{\mathrm{p}} \quad$ Class of porous media based on statically spherical inclusions, such that for each inclusion $\Psi_{\mathrm{p}}\left(\rho_{n}\right) R_{n}^{2}=K^{*}$.

$\mathrm{PM}_{\mathrm{V}} \quad$ Class of porous media based on kinematically spherical inclusions, such that for each inclusion $\Psi_{\mathrm{v}}\left(\rho_{n}\right) R_{n}^{2}=K^{*}$.

\footnotetext{
${ }^{1}$ E-mail: claude.boutin $@$ entpe.fr
} 
$R \quad$ External radius of the inclusion.

$R_{i} \quad$ Radius of the solid sphere in the inclusion.

$\mathbf{V}^{\mathrm{e}}$ Homogeneous velocity in the Darcy medium related to $\operatorname{grad}\left(P^{\mathrm{e}}\right)=\mathbf{e}_{z}$.

$\mathbf{v}_{\mathrm{p}}$ Statically continuous field such that on any inclusion boundary: $\left[\sigma_{p} \cdot \mathbf{n}\right]=0$.

$\mathbf{v}^{\mathrm{p}}$ Exact velocity field in medium $\mathrm{PM}_{\mathrm{p}}$.

$\mathbf{v}_{\mathrm{v}} \quad$ Kinematically continuous fields such that on any inclusion boundary: $\left[\mathbf{v}_{\mathrm{v}}\right]=0$.

$\mathbf{v}^{\mathrm{v}}$ Exact velocity field in medium $\mathrm{PM}_{\mathrm{v}}$.

\section{Greek symbols}

$\Phi_{\mathrm{Lp}}(\rho), \Phi_{\mathrm{Tp}}(\rho) \quad$ Idem as function $\Psi_{\mathrm{p}}(\rho)$ but for cylindrical inclusion and longitudinal ( $\mathrm{L}_{\mathrm{L}}$ ) or transverse (T) flow.

$\Phi_{\mathrm{Lv}}(\rho), \Phi_{\mathrm{v}}(\rho) \quad$ Idem as function $\Psi_{\mathrm{v}}(\rho)$ but for cylindrical inclusion and longitudinal ( $\mathrm{L}$ ) or transverse (T) flow.

$\rho$

Ratio of internal and external radii of the inclusion.

$\Psi_{\mathrm{p}}(\rho)$

Function related to the static assumption for spherical inclusion.

$\Psi_{\mathrm{v}}(\rho)$

Function related to the kinematic assumption for spherical inclusion.

$\varpi_{\mathrm{p}}$

Exact velocity in the fluid shell of the inclusion $I(R, \rho)$ under static conditions at the external boundary.

$\varpi_{\mathrm{v}}$

Exact velocity in the fluid shell of the inclusion $I(R, \rho)$ under kinematic conditions at the external boundary.

Note

For fibrous porous media the same notations are used with the indices ${ }_{\mathrm{L}}$ and ${ }_{\mathrm{T}}$ for longitudinal and transverse flow.

\section{Introduction}

The permeability of porous materials is a physical parameter which is considered in various domains of mechanics, such as soil mechanics, petroleum engineering, acoustics of noise absorbing materials, moulding of fibre reinforced composites, etc. Predicting the permeability of a given medium is then of great interest and a great number of studies address the identification and the characterisation of this parameter.

With this aim, various methods were applied and have contributed widely to the clarification and the understanding of the mechanisms linked with the Darcy coefficient. These are: the phenomenological thermodynamics-based approach such as developed by Biot (1941), the micro-macro approach using either the homogenisation of periodic media (HPM) (Auriault and Sanchez-Palencia, 1977), or, more rarely, the selfconsistent method (SCM) as investigated by Berdichevsky and Cai (1993), and finally numerical simulations as developed, for example, by Sangani and Acrivos (1982). However, despite the fact that the physics of the phenomenon is well known, the question of a simple assessment of the permeability remains an open-ended problem.

In this paper we focus on this topic by using both HPM and SCM.

One of the advantages of HPM is to rigorously derive the macroscopic Darcy law from the Navier-Stokes equation at the pores scale. It also gives the theoretical expression of the Darcy tensor, whatever the periodic microstructure. However, the permeability value can only be obtained through computer simulations due to 
the significant complexity of the flow through the pores. This requires performing a number of simulations for identifying approximate relationships between permeability and microstructure. Moreover, for technical computing reasons, only rather simple microstructures can actually be investigated. Nevertheless the HPM constitutes an excellent guideline for studying physical phenomena at both micro- and macro-scale.

The interest of SCM is to propose a permeability value from analytical solutions in simplified configurations. Contrary to HPM the microstructure is not identified with accuracy, and if the basic analytical solution is correct, the application of the result to real porous media is generally conjectured (Christensen and Lo, 1979) for the case of elastic composites. In addition, in the case of the Darcy law, an additional difficulty arises due to the difference in the nature of governing equations in the fluid - vectorial balance equation with tensorial variables - and in the equivalent conjectured Darcy medium - scalar balance equation with vectorial variables. This led various authors to propose various permeability values based on the assumptions made for basic solutions (Berdichevsky and Cai, 1993;Tarnow, 1996).

This paper aims at combining the HPM and SCM approaches in order to obtain rigorous results for classes of porous media with an explicit description of their microstructures.

The paper is divided in four parts. In section 2 we review the derivation of the macroscopic description of the flow of a viscous fluid through porous media using the HPM method. Section 3 is devoted to the same problem using SCM and the basic principles identified with HPM. It is shown that two values of permeability can be conjectured. The interpretation of these results is treated in section 4 . We obtain exact bounds for the permeability of various classes of porous media constituted by fixed arrays of spherical grains - or cylindrical fibres - surrounded by a concentric fluid shell, the whole filling all the space. The result depends on both grain size distribution and fluid shell size distribution, and is valid for random or periodic spatial distribution.

\section{From Navier-Stokes to Darcy law using HPM}

\subsection{Homogenisation principles of periodic media}

Let us briefly review the basic principles of HPM. The homogenisation method of periodic structures is an asymptotic two-variable method (Sanchez-Palencia, 1980). The two well distinct macro-length, $L$, and microlength, $l$, led us to use two space variables, $x$ describing variations at the macroscopic scale, and $y$ the variations at the microstructure level. The small parameter $\varepsilon$ is the scale ratio:

$$
\varepsilon=l / L, \quad y=\varepsilon^{-1} x .
$$

The two space variables transform the common spatial derivatives into? $\partial_{x}+\varepsilon^{-1} \partial_{y}$. Due to the different orders of magnitude introduced by $\varepsilon$, any quantity $q$ is expressed in the form of asymptotic expansions in powers of $\varepsilon$ :

$$
q(x, y)=\Sigma \varepsilon^{j} q^{j}(x, y) \quad \text { with: } \mathrm{O}\left(q^{j} / q^{0}\right)=1 .
$$

The microperiodicity of the medium induces the same periodicity for the terms $q^{j}$, according to variable $y$.

The process consists of introducing expansions in the scaled equations governing physical phenomena at local scale (using powers of $\varepsilon$ for expressing the order of magnitude of the various quantities), then in identifying the terms of same power in $\varepsilon$, and finally in solving the problems obtained in series. 


\subsection{Darcy law}

The macroscopic description of the quasi-static laminar flow of a Newtonian viscous fluid through a nondeformable porous matrix was extensively studied by Auriault and Sanchez-Palencia (1977) in which the Darcy law was derived using the HPM approach. Nevertheless it is necessary to expose and comment the main steps and teachings of this approach prior to proceeding with the reasoning.

\subsubsection{The governing equations}

Considering the above assumptions, the set of equations governing the fluid motion in porous cell $\Omega$ (figure 1), where $\sigma$ is the stress in the fluid, $\sigma_{\mathrm{s}}$ the stress in the infinitely rigid porous solid, $p$ the pressure, $\mathbf{v}$ the velocity, $\mathbf{D}$ the rate of strain, and $\mu$ the viscosity, can be written:

In fluid $\Omega_{\mathrm{f}}$

$$
\begin{array}{ll}
\text { Incompressibility } & \operatorname{div}(\mathbf{v})=0, \\
\text { Viscous behaviour } & \sigma=-p \mathbf{I}+2 \mu \mathbf{D}(\mathbf{v}), \\
\text { Navier-Stokes equation } & \operatorname{div}(\sigma)=-\operatorname{grad}(p)+\mu \Delta(\mathbf{v})=\mathbf{0} .
\end{array}
$$

At fluid-solid interface $\Gamma$

$$
\begin{array}{ll}
\text { Adherence condition } & \mathbf{v}=0 . \\
\text { Stress continuity } & \sigma_{\mathrm{s}} \cdot \mathbf{n}=\sigma . \mathbf{n} .
\end{array}
$$

In solid $\Omega_{\mathrm{s}}$

$$
\text { Equilibrium } \operatorname{div}\left(\sigma_{\mathrm{s}}\right)=\mathbf{0} \text {. }
$$

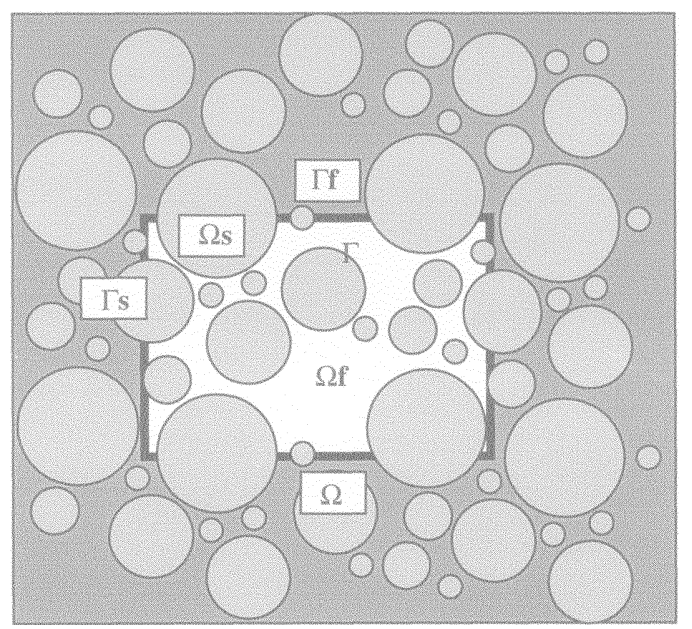

Figure 1. The periodic cell $\Omega$ of a porous medium. $\Omega_{\mathrm{f}}$ and $\Omega$ are, respectively, the volume of the fluid and the volume of the solid in the cell; $\Gamma$ is the fluid-solid interface inside $\Omega ; \Gamma_{\mathrm{f}}$ and $\Gamma_{\mathrm{S}}$ are the fluid and the solid interface at the boundary of $\Omega$. 


\subsubsection{Scaling}

The pressure gradient, which evolves at the macroscopic scale, is balanced by the viscous forces, which - as velocity - vary at the pores scale. This leads to the following estimations:

$$
\operatorname{grad}(p)=\mathrm{O}(p / L), \quad \mu \Delta(v)=\mathrm{O}\left(\mu v / l^{2}\right)
$$

so that:

$$
\mathrm{O}(p / L)=\mathrm{O}\left(\mu v / l^{2}\right) .
$$

Then, using $L$ as a reference length, the Navier-Stokes scaled equation, and the scaled expression of the stress are:

$$
\begin{gathered}
-\operatorname{grad}(p)+\varepsilon^{2} \mu \Delta(\mathbf{v})=0, \\
\sigma=-p \mathbf{I}+\varepsilon^{2} \mu \mathbf{D}(\mathbf{v}) .
\end{gathered}
$$

With these scaled equations (others are unchanged), we can proceed to the solving of the problems posed on the cell, all the quantities being $\Omega$-periodic according to variable $y$.

\subsubsection{Solution}

The first problem to be solved is $\left(5-\varepsilon^{-1}\right)$

$$
\operatorname{div}_{y}\left(\sigma^{0}\right)=-\operatorname{grad}_{y}\left(p^{0}\right)=0
$$

the solution of which is:

$$
\sigma^{0}=-p^{0}(x) \mathbf{I}=-P \mathbf{I}
$$

The second set of equations to be solved is $\left(5-\varepsilon^{0}\right),\left(1-\varepsilon^{-1}\right),\left(2-\varepsilon^{0}\right)$

$$
\begin{aligned}
& \operatorname{div}_{y}\left(\sigma^{1}\right)+\operatorname{div}_{x}\left(\sigma^{0}\right)=-\operatorname{grad}_{y}\left(p^{1}\right)-\operatorname{grad}_{x}(P)+\mu \Delta_{y}\left(\mathbf{v}^{0}\right)=\mathbf{0}, \\
& \operatorname{div}_{y}\left(\mathbf{v}^{0}\right)=0, \\
& \mathbf{v}^{0}=\mathbf{0} .
\end{aligned}
$$

The solution of this linear problem with the forcing term $\operatorname{grad}_{x}(P)$, is written in the form:

$$
v_{j}^{0}(x, y)=-\left[k_{j}^{i}(y) / \mu\right] \operatorname{grad}_{x}(P)_{i}, \quad p^{1}(x, y)=-\left[\pi^{i}(y)\right] \operatorname{grad}_{x}(P)_{i}+p^{1}(x),
$$

where $\mathbf{k}^{i}(y) / \mu$ and $\pi^{i}(y)$ are the particular velocity and pressure solution for unit macroscopic pressure gradient in direction $\mathbf{e}^{i},\left(\operatorname{grad}_{x}(P)=\mathbf{e}^{i}, i=1,2,3\right)$. Both $\mathbf{k}^{i}$ and $\pi^{i}$ only depend on the geometry of the pores with a rough order of magnitude of $\mathrm{O}\left(\mathbf{k}^{i}\right)=l^{2}, \mathrm{O}\left(\pi^{i}\right)=l$. Finally the macroscopic description is obtained by using $\left(1-\varepsilon^{0}\right),\left(2-\varepsilon^{1}\right)$ :

$$
\begin{aligned}
& \operatorname{div}_{y}\left(\mathbf{v}^{1}\right)+\operatorname{div}_{x}\left(\mathbf{v}^{0}\right)=0, \\
& \mathbf{v}^{1}=\mathbf{0} .
\end{aligned}
$$

Due to the periodicity of $v^{1}$, it remains after integration on the fluid volume of the cell:

$$
\operatorname{div}_{x}\left(\int_{\Omega_{f}} \mathbf{v}^{0}\right)=0 .
$$


Thus noting $\mathbf{V}=(1 / \Omega) \int_{\Omega_{f}} \mathbf{v}^{0} \mathrm{~d} \omega$, the Darcy law takes the form:

$$
\operatorname{div}_{x}(\mathbf{V})=0, \quad \mathbf{V}=-(\mathbf{K} / \mu) \operatorname{grad}_{x}(P) \quad \text { with: } K_{j}^{i}=(1 / \Omega) \int_{\Omega_{f}} k_{j}^{i}(y) \mathrm{d} \omega .
$$

\subsection{Comments}

\subsubsection{From micro to macro variables}

The quantities, which appear in the macroscopic description, are related to the local quantities in the pores:

- the macroscopic velocity, $\mathbf{V}$, is the average of the microscopic velocity in the cell.

- pressure $P$ is exactly given by the pore pressure. More precisely, this pressure is the stress of the zero order, which is constant in the fluid.

- as shown by Auriault and Sanchez-Palencia (1977), the intrinsic permeability tensor K, obtained by averaging the particular solutions $\mathbf{k}^{i}$, is directly related to the local viscous dissipation through:

$$
K_{j}^{i}=(2 / \Omega) \int_{\Omega_{\mathrm{f}}} \mathbf{D}\left(\mathbf{k}^{i}\right): \mathbf{D}\left(\mathbf{k}^{j}\right) \mathrm{d} \omega
$$

or

$$
2 \mu(1 / \Omega) \int_{\Omega_{\mathrm{f}}} \mathbf{D}\left(\mathbf{v}^{0}\right): \mathbf{D}\left(\mathbf{v}^{0}\right) \mathrm{d} \omega=\operatorname{grad}_{x}(P) .(\mathbf{K} / \mu) \cdot \operatorname{grad}_{x}(P)=-\mathbf{V} \cdot \operatorname{grad}_{x}(P) .
$$

In other words, the dissipation, which occurs on the elementary representative cell, is identical to that which would occur in the same volume of the equivalent Darcy medium. This demonstrates the energy consistency of the micro-macro passage using the HPM.

Conversely, the Darcy law obliterates the information from the pore level:

- the stress of the first order, $\sigma^{1}=-p^{1} \mathbf{I}+2 \mu \mathbf{D}_{y}\left(\mathbf{v}^{0}\right)$, which exists and varies at the local scale, disappears,

- only the average value of local velocity is known. Thus no simple relation can be a priori proposed to relate punctually the micro-velocity and the Darcy flow.

\subsubsection{Overall equilibrium of the cell}

An important point is that the Darcy law is obtained from the compatibility equation of the fluid mass balance. Since there is a stress transfer from the fluid to the skeleton, no compatibility equation can be obtained for the momentum balance on the fluid only. However a momentum compatibility equation can be established for both phases. Equations $\left(5-\varepsilon^{0}\right),(3-\varepsilon),\left(4-\varepsilon^{0}\right)$ give the following set:

$$
\begin{aligned}
& \operatorname{div}_{y}\left(\sigma^{1}\right)-\operatorname{grad}_{x}(P)=\mathbf{0}, \\
& \sigma_{\mathrm{s}}^{1} \cdot \mathbf{n}=\sigma^{1} \cdot \mathbf{n}, \\
& \operatorname{div}_{y}\left(\sigma_{\mathrm{s}}^{1}\right)+\operatorname{div}_{x}\left(\sigma_{\mathrm{s}}^{0}\right)=0 .
\end{aligned}
$$

Notice that, according to the periodicity of the variables, we get:

$$
\int_{\Gamma_{\mathrm{s}}} \sigma_{\mathrm{s}}^{1} \cdot \mathbf{n} \mathrm{d} s+\int_{\Gamma_{\mathrm{f}}} \sigma^{1} \cdot \mathbf{n} \mathrm{d} s=0 .
$$


This important equality expresses the fact that, on average, the stresses of the first order (and also highest) are self-equilibrated in the cell. In the particular case studied later on, where the solid is isolated in the cell, the equality reduces to:

$$
\int_{\Gamma_{\mathrm{f}}} \sigma^{1} \cdot \mathbf{n} \mathrm{d} s=0
$$

Integrating each balance equation over its own domain, the contributions of $\sigma_{\mathrm{s}}^{1}$ and $\sigma^{1}$ disappear by continuity so that we get for the overall momentum balance:

$$
\int_{\Omega_{\mathrm{s}}} \operatorname{div}_{x}\left(\sigma_{\mathrm{s}}^{0}\right) \mathrm{d} \omega=\int_{\Omega_{\mathrm{f}}} \operatorname{grad}_{x}(P) \mathrm{d} \omega
$$

This means that the pressure gradient in the fluid is counterbalanced by stresses in the solid skeleton.

To conclude this section, the HPM has given:

- the nature of the macroscopic behaviour (Darcy law),

- the description of local phenomena (flow governed by the Navier-Stokes equations),

- the effective relationship between local phenomena and macro quantities.

Nevertheless the HPM method does not provide a simple approach for obtaining an explicit expression of the Darcy coefficient. The next section is aimed at introducing the information obtained by HPM in the selfconsistent approach for assessing the permeability of a class of porous media.

\section{Conjectured permeability by SCM using spherical fluid-solid inclusions}

\subsection{Principles of the self-consistent method}

As exposed in the introduction, the self-consistent method provides a way to conjecture the effective macroscopic coefficients of heterogeneous media. Following Hashin (1968), the steps of this approach developed for various phenomena, are:

- assume the macroscopic behaviour, the coefficients of which being to determined,

- consider a (or various) complex (or simple) inclusion(s) embedded in such medium,

- solve the basic problem under homogeneous boundary conditions (applied at the bounds of the infinite medium),

- express energy equivalence between the whole of the representative inclusions and the equivalent medium,

- deduce from this relation the value of the macroscopic coefficients.

The application of SCM to the derivation of Darcy coefficient was recently investigated by Berdichevsky and Cai (1993) for fibrous materials. Other authors use the same 'philosophy' as SCM, but, instead of the energy consistency, additional kinematic assumptions are introduced for determining the basic solution. 


\subsubsection{Generic inclusion}

Concerning inclusion $I$ (volume $\Omega_{I}$, boundary $\partial \Omega_{I}$ ), we treated the case of a solid sphere (radius $R_{i}$, volume $\Omega_{\mathrm{s}}$ ) surrounded by a concentric spherical shell filled with a viscous Newtonian fluid of external radius $R$ and volume $\Omega_{\mathrm{f}}=\Omega_{I}-\Omega_{\mathrm{s}}$ (figure 2 ).

As usual in the self-consistent approach we considered two infinite media A and B (figure 3). Medium A is constituted by a homogeneous equivalent medium, whereas medium B corresponds to the same medium in which a given volume has been removed and replaced by composite inclusion $I$. The main idea was to compare both media, when A is submitted to homogeneous conditions (unit pressure gradient):

$$
\operatorname{grad}\left(P^{\mathrm{e}}\right)=\mathbf{e}_{z} \quad \text { so that: } \quad \mathbf{V}^{\mathrm{e}}=-(K / \mu) \mathbf{e}_{z}
$$

and when boundary conditions applied to whole structure B (equivalent medium + composite inclusion) tend to (7) at infinity.

\subsubsection{General expression of the fields}

Because of the spherical symmetry of the structure, and the privileged direction introduced by the pressure gradient at infinity, we used the spherical co-ordinates $(r, \theta, \phi)$ oriented as described in figure $2(\theta=0$ corresponds to $\mathbf{e}_{r}=\mathbf{e}_{z}$ ). The governing equations in both domains are (uppercase variables concern the Darcy medium and lowercase variables the fluid in the inclusion):

$$
\begin{array}{lll}
\text { - in the Darcy medium: } r>R & \operatorname{div}(\mathbf{V})=0 ; & \mathbf{V}=-(K / \mu) \operatorname{grad}(P), \\
\text { - in the fluid shell: } R>r>R_{i} & \operatorname{div}(\mathbf{v})=0 ; & -\operatorname{grad}(p)+\mu \Delta(\mathbf{v})=\mathbf{0} .
\end{array}
$$

According to the symmetry of the problem, the solutions are independent of $\phi$, and are considered in the following form:

$$
\begin{aligned}
& \text { Darcy medium: } r>R \\
& \text { Fluid shell: } R>r>R_{i} \\
& \mathbf{V}=\left\{\begin{array}{l}
\mathrm{V}_{r}=-(K / \mu) F(r) \cos (\theta), \\
V_{\theta}=(K / \mu) G(r) \sin (\theta), \\
V_{\phi}=0,
\end{array}\right. \\
& \mathbf{v}=\left\{\begin{array}{l}
v_{r}=-(K / \mu) f(r) \cos (\theta), \\
v_{\theta}=(K / \mu) g(r) \sin (\theta), \\
v_{\phi}=0,
\end{array}\right. \\
& P=H(r) \cos (\theta) . \\
& p=K h(r) \cos (\theta) \text {. }
\end{aligned}
$$

In the fluid, the components of the tensor of the rate of deformation are ('stands for $d / d r$ ):

$$
\begin{aligned}
& \mathrm{D}_{r r}=(-K / \mu) f^{\prime}(r) \cos (\theta), \\
& \mathrm{D}_{\theta \theta}=\mathrm{D}_{\phi \phi}=(-K / \mu)\{[f(r)-g(r)] / r\} \cos (\theta), \\
& \mathrm{D}_{r \theta}=(-K / \mu)\left\{g^{\prime}(r) / 2+[g(r)-f(r)] / 2 r\right\} \sin (\theta) .
\end{aligned}
$$

Introducing these functions into equations (8), (9) led after algebraic calculations to the following generic expressions which (for $F, G, H$ ) tend to the boundary condition (7) at infinity:

$$
\begin{array}{lll}
H(r)=\left[\alpha(R / r)^{3}+1\right] r, & h(r)=\left[-2 b(R / r)-10 d(R / r)^{-2}\right] / r, \\
F(r)=-2 \alpha(R / r)^{3}+1, & & f(r)=2 a(R / r)^{3}+2 b(R / r)+c+d(R / r)^{-2}, \\
G(r)=-\alpha(R / r)^{3}+1, & & g(r)=-a(R / r)^{3}+b(R / r)+c+2 d(R / r)^{-2} .
\end{array}
$$


Two convenient relations will be used in the following:

$$
h+f^{\prime}+2 g^{\prime}=-(6 b / R)(R / r)^{2}, \quad f^{\prime}=-2(f-g) / r .
$$

Hence the problem involves five parameters $(a, b, c, d, \alpha)$ and unknown $K$. To determine them we have now to specify the conditions these fields have to fulfil.

\subsection{Conditions for the fields}

\subsubsection{The argument of energy}

Let us now express the energy equivalence between both loaded media A and B. For the problem studied, we have to determine the dissipated powers, $E_{\mathrm{A}}$ and $E_{\mathrm{B}}$ in medium $\mathrm{A}$ and B. For simplicity, these values are calculated over spherical domains of radius $R_{\infty} \gg R$.

In medium $\mathrm{A}$, the pressure gradient and velocity are constant so that:

$$
2 E_{\mathrm{A}}=\int_{\mathrm{A}}-\operatorname{grad}\left(P^{\mathrm{e}}\right) \cdot \mathbf{V}^{\mathrm{e}} \mathrm{d} \omega=(K / \mu) R_{\infty}^{3} 4 \pi / 3 .
$$

In medium B we have to separate the external homogeneous domain and the inclusion.

$$
2 E_{\mathrm{B}}=\int_{\mathrm{B}-I}-\operatorname{grad}(P) \cdot \mathbf{V} \mathrm{d} \omega+2 E_{I} \quad \text { where: } 2 E_{I}=2 \mu \int_{\Omega_{\mathrm{f}}} \mathbf{D}(\mathbf{v}): \mathbf{D}(\mathbf{v}) \mathrm{d} \omega .
$$

With the divergence theorem and the flow incompressibility, $E_{\mathrm{B}}$ is transformed into:

$$
2 E_{\mathrm{B}}=\int_{\partial(\mathrm{B}-I)}-P \mathbf{V} \cdot \mathbf{n} \mathrm{d} s+2 E_{I} .
$$

Using the expressions of $P$ and $\mathbf{V}$ given in the above section, we get:

$$
2 E_{\mathrm{B}}=4 \pi / 3(K / \mu)\left\{\left[\alpha\left(R / R_{\infty}\right)^{3}+1\right]\left[-2 \alpha\left(R / R_{\infty}\right)^{3}+1\right] R_{\infty}^{3}-[\alpha+1][-2 \alpha+1] R^{3}\right\}+2 E_{I} .
$$

Now, both medium A and B are energetically equivalent if:

$$
E_{\mathrm{A}}=E_{\mathrm{B}} \quad \text { or } \quad E_{\mathrm{B}} / E_{\mathrm{A}}=1
$$

i.e.:

$$
\left[1+\alpha\left(R / R_{\infty}\right)^{3}\right]\left[1-2 \alpha\left(R / R_{\infty}\right)^{3}\right]-[\alpha+1][-2 \alpha+1]\left(R / R_{\infty}\right)^{3}+2\left[E_{i} /(K / \mu) R^{3} 4 \pi / 3\right]\left(R / R_{\infty}\right)^{3}=1
$$

Whatever could be the characteristics of the inclusion, such a relation is obviously valid when $R / R_{\infty} \rightarrow 0$, for the simple reason that the volume of the inclusion becomes negligible.

However, we are looking for a specific situation which optimises the energetic equivalence. This imposes the identification of the parameters for which the equality is reached as rapidly as possible when $R / R_{\infty} \rightarrow 0$. Organising relation (10) in decreasing powers of $R / R_{\infty}$ lead successively to cancel: 
- the term in $\left(R / R_{\infty}\right)^{3}$, which gives: $-\alpha-[\alpha+1][-2 \alpha+1]+2\left[E_{i} /(K / \mu) R^{3} 4 \pi / 3\right]=0$,

- the remaining term in $\left(R / R_{\infty}\right)^{6}$, which gives: $-2 \alpha^{2}=0$, i.e. $\alpha=0$.

Therefore the preceding relation reduces to:

$$
(K / \mu) R^{3} 4 \pi / 3=2 E_{I}
$$

It should be noted that with these optimised conditions, the energy equivalence is satisfied whatever the radius $R_{\infty}>R$. This result has two consequences:

First, $\alpha=0$, means that the interactions between the equivalent medium and the representative inclusion are reduced to zero. In other terms, the fields in the Darcy medium in absence (medium A) or in presence of the inclusion (medium B) are exactly the same, i.e., for $r>R$ :

$$
\operatorname{grad}(P)=\operatorname{grad}\left(P^{\mathrm{e}}\right)=\mathbf{e}_{z} \quad \text { and: } \quad \mathbf{V}=\mathbf{V}^{\mathrm{e}}=-(K / \mu) \mathbf{e}_{z} .
$$

Second, $(K / \mu) R^{3} 4 \pi / 3=2 E_{I}$ means that the energy equivalence for both medium $\mathrm{A}$ and $\mathrm{B}$ simply reduces to the equality of the dissipated power in the inclusion on the one hand, and in a same volume of the equivalent Darcy medium on the other hand. We will come back to this condition later on.

\subsubsection{Conditions for the fields in the inclusion}

In accordance with the adherence condition, the velocity at the fluid-solid interface must vanish. Therefore:

$$
\begin{aligned}
& f\left(R_{i}\right)=0, \\
& g\left(R_{i}\right)=0 .
\end{aligned}
$$

Moreover, the average velocity in the representative inclusion must equal the velocity in the equivalent Darcy medium. We get:

$$
\begin{aligned}
\left(1 / \Omega_{I}\right) \int_{\Omega_{\mathrm{f}}} \mathbf{v} \mathrm{d} \omega & =\left(1 / \Omega_{I}\right) \int_{\partial \Omega_{\mathrm{I}}} \mathbf{r} .\left(\mathbf{v} . \mathbf{e}_{r}\right) \mathrm{d} s+0=\left(2 \pi / \Omega_{I}\right) \int r v_{r}\left[\sin (\theta) \mathbf{e}_{x}+\cos (\theta) \mathbf{e}_{z}\right] \sin (\theta) R^{2} \mathrm{~d} \theta \\
& =-(K / \mu) f(R) \mathbf{e}_{z}+0=-(K / \mu) \mathbf{e}_{z}
\end{aligned}
$$

and therefore:

$$
f(R)=1 .
$$

Notice that since $\alpha=0$, with $f(R)=1$, the normal velocity in the fluid and in the equivalent Darcy medium is continuous at any point of the boundary:

$$
v_{r}(R)=V_{r}(R)=V_{r}^{\mathrm{e}} .
$$

This relation expresses the local mass balance at the boundary. Assuming this condition would have been another way to deduce $\alpha=0$ independently of the above energy considerations.

\subsubsection{Condition at the Inclusion - Darcy medium boundary: overall equilibrium}

This condition is less obvious than the previous one. The question of boundary conditions between a fluid and a porous medium was discussed in detail in Levy and Sanchez-Palencia (1975). However the analysis is 
only valid if the fluid domain is significantly larger than the pores, which is not the case in the considered condition.

The difficulty arises from the fact that the Darcy law doesn't integrate the momentum balance in the fluid. Since a part of the viscous stress is transferred to the skeleton, it is not possible to assume a priori the local continuity of the pressure or of the stress.

However, is has been proven using HPM (section 2.3) that the force exerted by the 'corrective' stresses i.e., stresses of order higher than one - applied at the boundary of the representative volume equals zero. This corresponds to the fact that the equilibrium of the ERV is satisfied on average by a stress of the zero order. It is physically acceptable to transpose this result to the present situation. The corrective stress, $\sigma^{\mathrm{c}}$, is given by the difference between the stress in the fluid $(\sigma)$ and the stress (opposite of pressure) in the Darcy medium $\left(-P \mathbf{I}=-P^{\mathrm{e}} \mathbf{I}\right)$ :

$$
\sigma^{\mathrm{c}}=\sigma+P^{\mathrm{e}} \mathbf{I}=[-p \mathbf{I}+2 \mu \mathbf{D}(\mathbf{v})]+P^{\mathrm{e}} \mathbf{I} \quad \text { and: } \quad \int_{\partial \Omega_{I}} \sigma^{\mathrm{c}} \cdot \mathbf{n} \mathrm{d} s=0
$$

i.e.:

$$
\int_{\partial \Omega_{I}} \sigma \cdot \mathbf{n} \mathrm{d} s=-\int_{\partial \Omega_{I}} P^{\mathrm{e}} \mathbf{n} \mathrm{d} s .
$$

In this latest form it clearly appears that this condition is an 'averaged' continuity equation between the stress in the fluid and the pressure in the Darcy medium, or an overall equilibrium condition of the inclusion. Introducing the components of the stress tensor gives:

$$
\int_{\partial \Omega_{I}} \sigma^{\mathrm{c}} \cdot \mathbf{e}_{r} \mathrm{~d} s=\int_{\partial \Omega_{I}}\left[P^{\mathrm{e}}-p+2 \mu \mathrm{D}_{r r}(\mathbf{v})\right] \mathbf{e}_{r}+2 \mu \mathrm{D}_{r \theta}(\mathbf{v}) \mathbf{e}_{\theta} \mathrm{d} s=0
$$

i.e.:

$$
\int_{\partial \Omega_{I}}\left\{\left[P^{\mathrm{e}}-p+2 \mu \mathrm{D}_{r r}(\mathbf{v})\right]\left[\sin (\theta) \mathbf{e}_{x}+\cos (\theta) \mathbf{e}_{z}\right]+2 \mu \mathrm{D}_{r \theta}(\mathbf{v})\left[\cos (\theta) \mathbf{e}_{x}-\sin (\theta) \mathbf{e}_{z}\right]\right\} \mathrm{d} s=0 .
$$

Due to the symmetry of the problem, the $\mathbf{e}_{x}$ component vanishes and only a single $\mathbf{e}_{z}$ component-related equation remains:

$$
\int_{\partial \Omega_{I}}\left\{\left[P^{\mathrm{e}}-p+2 \mu \mathrm{D}_{r r}(\mathbf{v})\right] \cos (\theta)-2 \mu \mathrm{D}_{r \theta}(\mathbf{v}) \sin (\theta)\right\} \mathrm{d} s=0
$$

and, after calculations:

$$
R-K\left\{h(R)+2\left[f^{\prime}(R)+g^{\prime}(R)+(f(R)-g(R)) / R\right]\right\}=0 .
$$

\subsubsection{Back to the energy condition}

From the above results, we can proceed with the energy condition.

In the fluid shell, due to incompressibility, we get for the dissipated power:

$$
2 E_{I}=2 \mu \int_{\Omega_{I f}} \mathbf{D}(\mathbf{v}): \mathbf{D}(\mathbf{v}) \mathrm{d} \omega=\int_{\Omega_{I f}}[-p \mathbf{I}+2 \mu \mathbf{D}(\mathbf{v})]: \mathbf{D}(\mathbf{v}) \mathrm{d} \omega=\int_{\Omega_{I f}} \sigma: \mathbf{D}(\mathbf{v}) \mathrm{d} \omega
$$

which, using the divergence theorem and the adherence condition, is also equal to

$$
2 E_{I}=\int_{\partial \Omega_{I}}(\sigma \cdot \mathbf{n}) \cdot \mathbf{v} \mathrm{d} s .
$$


In the Darcy medium (A), the power dissipated in a volume identical to that of the inclusion takes the form:

$$
\int_{\Omega_{I}}-\operatorname{grad}\left(P^{\mathrm{e}}\right) \cdot \mathbf{V}^{\mathrm{e}} \mathrm{d} \omega=\int_{\partial \Omega_{I}}\left(P^{\mathrm{e}} \mathbf{V}^{\mathrm{e}} \cdot \mathbf{n}\right) \mathrm{d} s .
$$

However, as $\mathbf{V}^{\mathrm{e}}$ is constant and because of (14), we obtain successively:

$$
\int_{\partial \Omega_{I}}\left(P^{\mathrm{e}} \mathbf{V}^{\mathrm{e}} \cdot \mathbf{n}\right) \mathrm{d} s=\mathbf{V}^{\mathrm{e}} \cdot \int_{\partial \Omega_{I}}\left(P^{\mathrm{e}} \mathbf{n}\right) \mathrm{d} s=\mathbf{V}^{\mathrm{e}} \cdot \int_{\partial \Omega_{I}}(\sigma \cdot \mathbf{n}) \mathrm{d} s=\int_{\partial \Omega_{I}}(\sigma \cdot \mathbf{n}) \cdot \mathbf{V}^{\mathrm{e}} \mathrm{d} s .
$$

Therefore the equality of the two dissipated powers becomes:

$$
\int_{\partial \Omega_{I}}(\sigma \cdot \mathbf{n}) \cdot\left(\mathbf{v}-\mathbf{V}^{\mathrm{e}}\right) \mathrm{d} s=\int_{\partial \Omega_{I}}\left(\sigma \cdot \mathbf{e}_{r}\right) \cdot\left(\mathbf{v}-\mathbf{V}^{\mathrm{e}}\right) \mathrm{d} s=\int_{\partial \Omega_{I}}\left[\left(\sigma_{r r}\right)\left(v_{r}-V_{r}^{\mathrm{e}}\right)+\left(\sigma_{r \theta}\right)\left(v_{\theta}-V_{\theta}^{\mathrm{e}}\right)\right] \mathrm{d} s=0 .
$$

Finally, owing to the continuity of the normal velocity at the interface (13), it only remains:

$$
\int_{\partial \Omega_{I}}\left(2 \mu \mathrm{D}_{r \theta}\right)\left(v_{\theta}-V_{\theta}^{\mathrm{e}}\right) \mathrm{d} s=0
$$

According to the expressions of the fields, two alternatives are available at the boundary:

- either the tangential velocities are continuous.

$$
v_{\theta}(R)=V_{\theta}^{\mathrm{e}} \quad \text { and then: } \quad g(R)=1 .
$$

With $f(R)=1$, this necessarily leads to $f^{\prime}(R)=0$, so that $2 \mu \mathrm{D}_{r r}=0$. Then (15) becomes:

$$
R-K\left[h(R)+2 g^{\prime}(R)\right]=0 .
$$

- or the shear stress $\sigma_{r \theta}=2 \mu \mathrm{D}_{r \theta}$ vanishes uniformly, and therefore $\sigma . \mathbf{n}=\sigma_{r r} \mathbf{e}_{r}=P \mathbf{e}_{r}$ which enables to simplify (15):

$$
\begin{gathered}
D_{r \theta}=0 \quad \text { i.e.: } \quad g^{\prime}(R)+[f(R)-g(R)] / R=0, \\
\sigma_{r r}-P=0 \quad \text { i.e.: } \quad R-K\left[h(R)+2 f^{\prime}(R)\right]=0 .
\end{gathered}
$$

To summarise, we obtain two sets of five conditions (in addition to $\alpha=0$ ).

Three of them are common to both sets, i.e., the two conditions related to the adherence condition (11-r), (11- $\theta$ ), and the one expressing the continuity between normal velocity and Darcy flow (12). This latter, derived here from the energy condition, is usually directly assumed by the authors.

The two remaining are derived from combining the energy equivalence and the 'averaged' stress continuity or overall equilibrium condition. This last one is determined directly from a comparison with the HPM approach and has not been proposed up to now. It appears that two alternatives can be selected:

- either the micro-velocity fits the Darcy flow and neither the pressure nor the stress is continuous. This will be called the kinematic approach hereafter.

- or the shear stress vanishes and the stress is continuous with pressure in the Darcy medium. It will be called the static approach below. In this case we find the assumptions made by Berdichevsky and Cai (1993). The tangential micro-velocity is not continuous with the tangential Darcy flow. 
It should be noted that when using such an approach, the assumption of vanishing vorticity at the boundary of the cell - used in various studies (for example Tarnow (1996)) - does not appear naturally. This latter assumption does not meet the global equilibrium of the cell and seems to be an acceptable approximation only for very porous medium $\left(\Omega_{I \mathrm{~s}} / \Omega_{I} \rightarrow 0\right)$.

\subsection{Solutions}

Since no physical reasons enable us to discard the kinematic or the static hypothesis, the two approaches have to be treated.

\subsubsection{Kinematic approach: field $\varpi_{\mathrm{v}}$}

Under the kinematics assumption, the five conditions allow the determination of the five remaining parameters $\left(a, b, c, d, K_{\mathrm{v}}\right)$. Using the expression of functions $f, g, h$, and introducing the notation $\rho=R_{i} / R$ lead to a linear system (11-r), (11- $\theta),(12),(16),(17)$ :

$$
\begin{array}{ll}
2 a \rho^{-3}+2 b \rho^{-1}+c+d \rho^{2}=0, & v_{r}\left(R_{i}\right)=0, \\
-a \rho^{-3}+b \rho^{-1}+c+2 d \rho^{2}=0, & v_{\theta}\left(R_{i}\right)=0 \\
2 a+2 b+c+d=1, & v_{r}(R)=V_{r}^{\mathrm{e}} \\
-a+b+c+2 d=1, & v_{\theta}(R)=V_{\theta}^{\mathrm{e}} \\
+b=-R^{2} / 6 K_{\mathrm{v}}, & (17)
\end{array}
$$

The resolution of this set can be divided in two steps: first, the calculation using the adherence and kinematic conditions of parameters $(a, b, c, d)$ of field $\varpi_{\mathrm{v}}$, then the determination of the $\mathrm{K}_{\mathrm{v}}$ value. Finally we obtain:

$$
K_{\mathrm{v}} / R^{2}=\Psi_{\mathrm{v}}(\rho)=[(1-\rho) / \rho]\left[4-5 \rho(1+\rho)\left(1-\rho^{2}\right) /\left(1-\rho^{5}\right)\right] / 18
$$

and:

$$
a=-(\beta-1) / 18 \Psi_{\mathrm{v}}, \quad b=-1 / 6 \Psi_{\mathrm{v}}, \quad c=1-(5 \beta+4) / 18 \Psi_{\mathrm{v}}, \quad d=\beta / 18 \Psi_{\mathrm{v}},
$$

where:

$$
\beta=\left(1-\rho^{2}\right) /\left(1-\rho^{5}\right) .
$$

From expression (20) it can be proven that $K_{\mathrm{v}}$ is positive. Note that $\varpi_{\mathrm{v}}$ is the 'exact field' for the flow through the fluid shell under the imposed kinematic conditions at the external boundary $(r=R): \varpi_{\mathrm{v}}=-\left(K_{\mathrm{v}} / \mu\right) \mathbf{e}_{z}$.

\subsubsection{Static approach: field $\varpi_{\mathrm{p}}$}

From the static assumption, the five conditions (11-r), (11- $\theta),(18),(19),(12)$ can be written:

$$
\begin{array}{ll}
2 a \rho^{-3}+2 b \rho^{-1}+c+d \rho^{2}=0, & v_{r}\left(R_{i}\right)=0, \\
-a \rho^{-3}+b \rho^{-1}+c+2 d \rho^{2}=0, & v_{\theta}\left(R_{i}\right)=0, \\
2 a+0+0+d=0, & D_{r \theta}=0, \\
2 a+b+0+d=-R^{2} / 6 K_{\mathrm{p}}, & \sigma_{r r}(R)=P^{\mathrm{e}}(R), \\
2 a+2 b+c+d=1, & v_{r}(R)=V_{r}^{\mathrm{e}} .
\end{array}
$$


The treatment of this set gives:

$$
K_{\mathrm{p}} / R^{2}=\Psi_{\mathrm{p}}(\rho)=[(1-\rho) / \rho]\left[1-\left(1-\rho^{5}\right) /(1-\rho)\left(2 \rho^{5}+3\right)\right] / 3
$$

and:

$$
a=\rho^{2} / 6\left(2 \rho^{5}+3\right) \Psi_{\mathrm{p}}, \quad b=-1 / 6 \Psi_{\mathrm{p}}, \quad c=1-2 b, \quad d=-2 a .
$$

Here again it can be proven that $K_{\mathrm{p}}$ is positive. Note that $\varpi_{\mathrm{p}}$ is the 'exact field' for the flow through the fluid shell under the imposed static condition at the external boundary $(r=R): \sigma_{\mathrm{p}} \cdot \mathbf{n}=-P \mathbf{n}$, with $\operatorname{grad}(P)=\mathbf{e}_{z}$.

\subsubsection{Comparison of the kinematic and static values}

Expression (20) gives the intrinsic permeability of the Darcy medium kinematically consistent with the inclusion $I$ characterised by $\rho$ and $R$. Similarly, expression (21) gives the intrinsic permeability of the Darcy medium statically consistent with the inclusion $I$ characterised by $\rho$ and $R$. In such a medium of permeability $K_{\mathrm{v}}\left(\right.$ respectively $K_{\mathrm{p}}$ ) the inclusion is kinematically (respectively statically) neutral since its presence does not modify neither the average density of dissipated power nor the field in the Darcy medium, and the flow continuity (respectively stress continuity) is provided.

Figure 4 shows the variations of functions $\Psi_{\mathrm{v}}$ and $\Psi_{\mathrm{p}}$ versus $\rho$. The analysis of both functions shows that $\Psi_{\mathrm{p}}>\Psi_{\mathrm{v}}$ and their limit values when $\rho$ tends to 0 and to 1 are:

$$
\begin{array}{ll}
\rho \rightarrow 0, & \rho \rightarrow 1, \\
\Psi_{\mathrm{v}}(\rho) \rightarrow(2 / 9) \rho^{-1}-1 / 2, & \Psi_{\mathrm{v}}(\rho) \rightarrow(1-\rho)^{3} / 6, \\
\Psi_{\mathrm{p}}(\rho) \rightarrow(2 / 9) \rho^{-1}-1 / 3, & \Psi_{\mathrm{p}}(\rho) \rightarrow 2(1-\rho)^{3} / 3 .
\end{array}
$$

Then, for same values of $\rho$ and $R, K_{\mathrm{p}} / K_{\mathrm{v}} \rightarrow 1$ when $\rho \rightarrow 0$ (dilute solid concentration) whereas, when $\rho \rightarrow 1$ (dense solid concentration), $K_{\mathrm{p}}$ and $K_{\mathrm{v}}$ differ significantly since $K_{\mathrm{p}} / K_{\mathrm{v}} \rightarrow 4$.

Generally the determination of the equivalent parameter constitutes the ultimate step of a self-consistent approach. The obtained value is assumed to be the effective coefficient of the heterogeneous medium characterised by inclusions $I$.

However, the case of the Darcy law is specific for two main reasons. First the analysis leads to two different values which both seems physically acceptable. Second, contrary to other physical problems (elasticity, conductivity, etc.) the value of $K$ depends on the size of the inclusion (in addition to the usual dependence on the concentration of the constituents). It is then necessary to examine in which situations the above results can be applied. This point is discussed below.

\section{Interpretation bounds for permeability of classes of porous media}

\subsection{From neutral inclusions to classes of permeable media $\mathrm{PM}_{\mathrm{v}}, \mathrm{PM}_{\mathrm{p}}$}

Up to now we have considered the problem of finding an equivalent medium from a given inclusion. Let as examine the inverse question. Consider a Darcy medium of permeability $K^{*}$. We have seen above that, without changing the macroscopic description, a sphere of radius $R_{0}$ can be replaced by a neutral inclusion $I_{0}\left(\rho_{0}, R_{0}\right)$ kinematically consistent with $K^{*}$, i.e.:

$$
K^{*}=\Psi_{\mathrm{v}}\left(\rho_{0}\right) R_{0}^{2}
$$



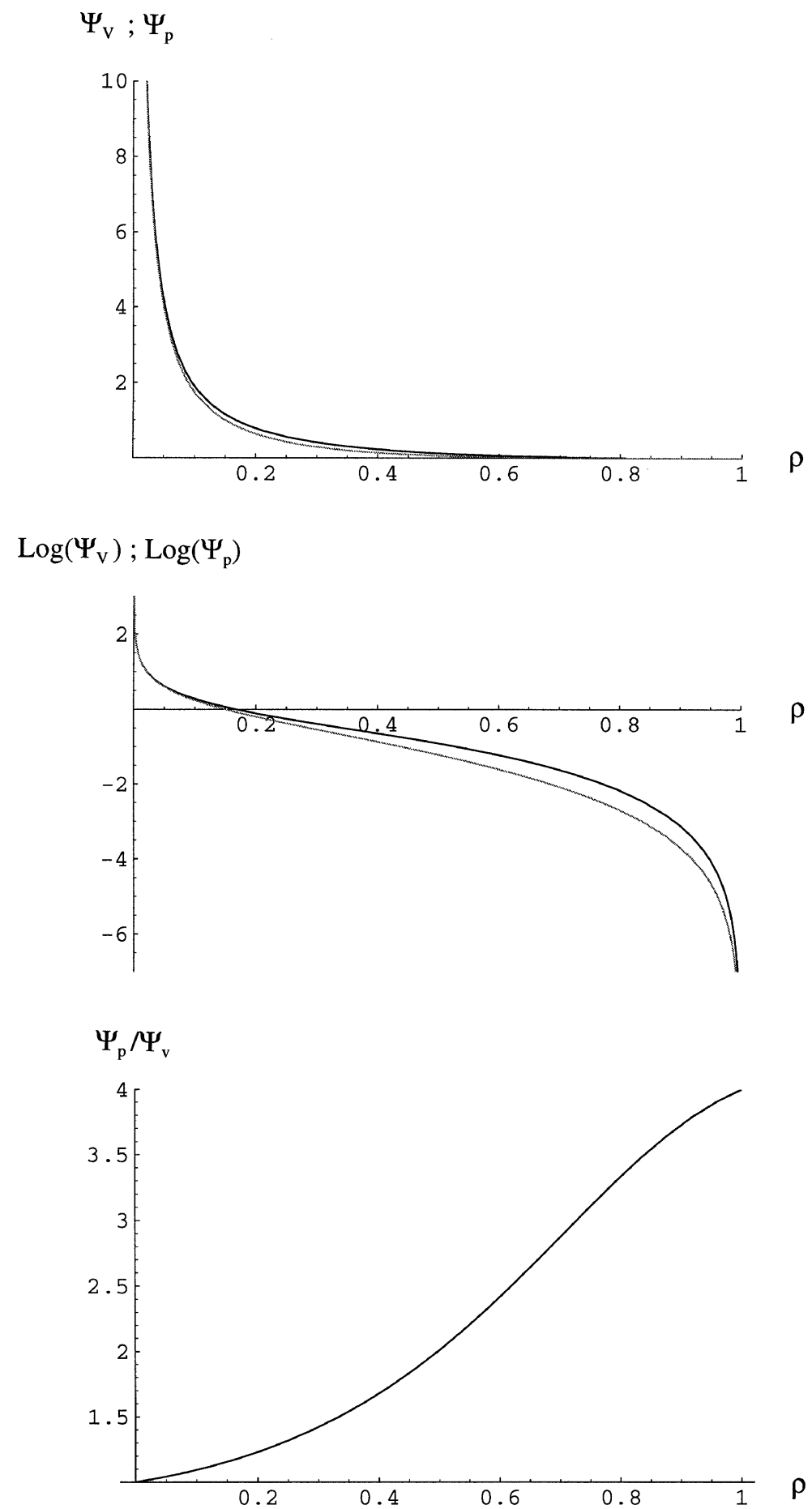

Figure 4. Spherical inclusions. Functions $\Psi_{\mathrm{p}}$ (straight line) and $\Psi_{\mathrm{v}}$ (dashed line). Top: $\Psi_{\mathrm{p}}$ and $\Psi_{\mathrm{v}}$ versus $\rho . \operatorname{Middle}: \log \left(\Psi_{\mathrm{p}}\right)$ and $\log \left(\Psi_{\mathrm{v}}\right)$ versus $\rho$ (Log decimal). Bottom: Ratio $\Psi_{\mathrm{p}} / \Psi_{\mathrm{v}}$ versus $\rho$. 


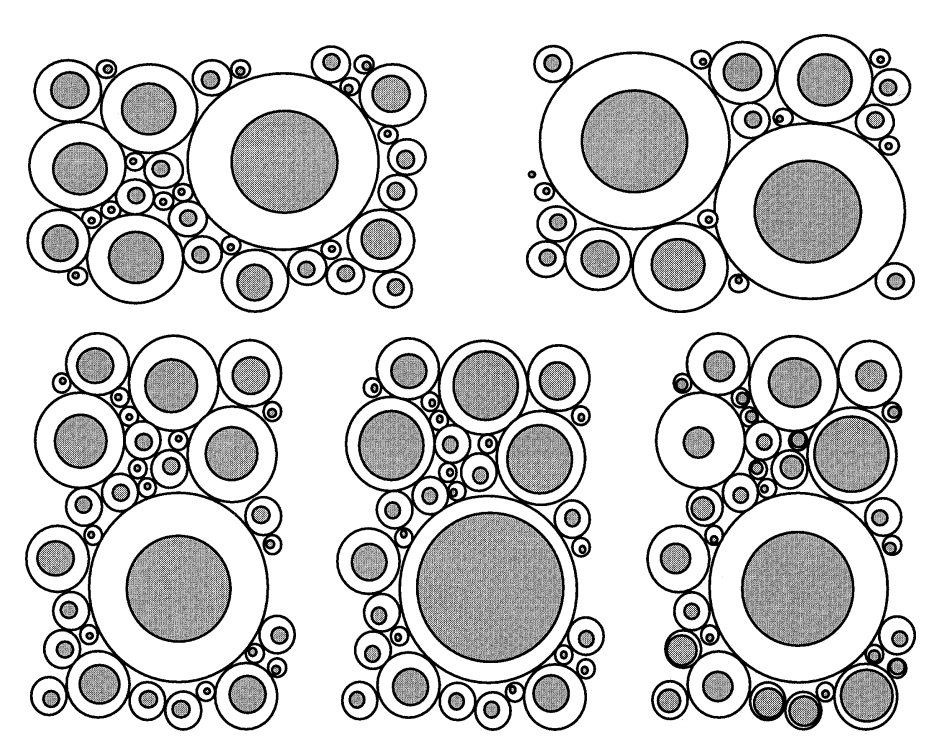

Figure 5. Classes of porous media (fixed arrays of particles) $\mathrm{PM}_{V}, \mathrm{PM}_{\mathrm{p}}$, PM. Top: Two examples of porous media of the same class $\mathrm{PM}_{\mathrm{V}}$ built with two sphere distributions. Each inclusion follows (20). Down: Porous media $\mathrm{PM}_{\mathrm{V}}, \mathrm{PM}_{\mathrm{p}}$ (each inclusion follows (21)) and PM built from the same sphere distribution as $\mathrm{PM}_{\mathrm{V}}$.

Due to the neutrality of $I_{0}\left(\rho_{0}, R_{0}\right)$, this operation can be repeated in any location (without superposition) to build a heterogeneous medium constituted by a permeable matrix in which identical neutral inclusions $I_{0}$ are inserted (periodically, randomly, etc.). If we want to tend to a more realistic medium, the spaces remaining between inclusions $I_{0}$ can be filled with smaller neutral inclusions $I_{1}$ of radius $R_{1}$. However to keep the energy consistency, $\rho_{1}$ must be such that:

$$
\Psi_{\mathrm{v}}\left(\rho_{1}\right) R_{1}^{2}=\Psi_{\mathrm{v}}\left(\rho_{0}\right) R_{0}^{2}=K^{*} .
$$

This process can be continued up to replacing the entire initial permeable matrix by a series of inclusions $I_{n}$ of radius $R_{N}$ where $\Psi_{\mathrm{v}}\left(\rho_{n}\right) R_{n}^{2}=K^{*}$. Note that as the function $\Psi_{\mathrm{v}}$ uniformly decreases with $\rho$, the solid concentration is reduced as $R_{n}$ decreases.

By this operation we built a class of porous media $\mathrm{PM}_{\mathrm{v}}$ (more exactly of fixed arrays of particles, see figure 5). The geometry of all these porous media is rather diversified since it includes numerous possible grain size distributions and arrangements, but, on the other hand, it is rather restricted by the imposed relation between the fluid shell and the grain size.

Similarly another class of porous media $\mathrm{PM}_{\mathrm{p}}$ based on statically neutral inclusions can be defined in order to obtain $\Psi_{\mathrm{p}}\left(\rho_{n}\right) R_{n}^{2}=K^{*}$.

Does value $K^{*}$ correspond to the intrinsic permeability of the two classes of porous media $\mathrm{PM}_{\mathrm{v}}$ and $\mathrm{PM}_{\mathrm{p}}$ ?

To answer this question we examine if the local fields determined by the self-consistent approach fit the exact field in the pores. By construction, the Navier-Stokes and the incompressibility equations as well as the adherence condition are fulfilled in the fluid of each inclusion. The difference lies in the boundary conditions:

As all the inclusions are in contact with other inclusions, the velocity and stress of the exact solution are continuous at the boundary of each inclusion. However, by construction, in the kinematic approach, the continuity of the micro-velocity is provided, but not the continuity of stresses. Conversely for the static approach, the continuity of stresses is ensured, but not the velocity continuity. 
This led us to consider that the actual permeability $K^{\mathrm{v}}$ and $K^{\mathrm{p}}$ of any media $\mathrm{PM}_{\mathrm{v}}$ (build in such way that for each inclusion $K_{\mathrm{v}}=K^{*}$ ) and media $\mathrm{PM}_{\mathrm{p}}$ (build in such way that for each inclusion $K_{\mathrm{p}}=K^{*}$ ) differs from $K^{*}$. This we will demonstrate hereafter.

\subsection{Kinematically and statically continuous fields for classes of permeable media PM}

Consider an arrangement of spheres of various radii $\left(R_{n}\right)$, filling the whole space. From that structure a wide class of media PM can be built by inserting a solid concentric spherical particle into each sphere. Among these media $\mathrm{PM}$, the two media $\mathrm{PM}_{\mathrm{v}}$ and $\mathrm{PM}_{\mathrm{p}}$ related to $K^{*}$ are obtained when the radius of the solid concentric spheres are such that: $\Psi_{\mathrm{v}}\left(\rho_{n}\right) R_{n}^{2}=K^{*}\left(\right.$ medium $\left.\mathrm{PM}_{\mathrm{v}}\right)$ or by $\Psi_{\mathrm{p}}\left(\rho_{n}\right) R_{n}^{2}=K^{*}\left(\right.$ medium $\left.\mathrm{PM}_{\mathrm{p}}\right)$.

In order to approximate the exact solution, let consider (for any porous medium PM) local fields such that the Navier-Stokes equation, the incompressibility and the adherence condition are fulfilled in the fluid shell volume of any sphere. Among these fields we distinguish ([ ] stands here for the jump through the boundary):

- kinematically continuous fields $\mathbf{v}_{\mathrm{v}}$ such that on any inclusion boundary: $\left[\mathbf{v}_{\mathrm{v}}\right]=0$,

- statically continuous field $\mathbf{v}_{\mathrm{p}}$ such that on any inclusion boundary: $\left[\sigma_{\mathrm{p}} \cdot \mathbf{n}\right]=0$,

- the exact solution, $\mathbf{v}$, which is the only field statically and kinematically continuous.

For the whole inclusions, we introduce the coupled dissipated power between any kinematically and statically continuous fields by:

$$
2 E_{\mathrm{c}}\left(\mathbf{v}_{\mathrm{p}}, \mathbf{v}_{\mathrm{v}}\right)=(2 \mu) \sum_{I} \int_{\Omega_{I}} \mathbf{D}\left(\mathbf{v}_{\mathrm{p}}-\mathbf{v}_{\mathrm{v}}\right): \mathbf{D}\left(\mathbf{v}_{\mathrm{p}}-\mathbf{v}_{\mathrm{v}}\right) \mathrm{d} \omega .
$$

By construction, $E_{\mathrm{c}}$ is positive. Its minimum value (zero) is reached for fields $\mathbf{v}_{\mathrm{p}}=\mathbf{v}_{\mathrm{v}}$ which is only possible for the exact solution $\mathbf{v}$. Therefore:

$$
\forall \mathbf{v}_{\mathrm{p}}, \mathbf{v}_{\mathrm{v}} \quad 2 E_{\mathrm{c}}\left(\mathbf{v}_{\mathrm{p}}, \mathbf{v}_{\mathrm{v}}\right) \geqslant 0 .
$$

Developing the expression of $2 E_{\mathrm{c}}\left(\mathbf{v}_{\mathrm{p}}, \mathbf{v}_{\mathrm{v}}\right)$ leads to:

$$
(2 \mu) \sum_{I}\left\{\int_{\Omega_{I \mathrm{f}}} \mathbf{D}\left(\mathbf{v}_{\mathrm{p}}\right): \mathbf{D}\left(\mathbf{v}_{\mathrm{p}}\right) \mathrm{d} \omega+\int_{\Omega_{I \mathrm{f}}} \mathbf{D}\left(\mathbf{v}_{\mathrm{v}}\right): \mathbf{D}\left(\mathbf{v}_{\mathrm{v}}\right) \mathrm{d} \omega\right\} \geqslant(4 \mu) \sum_{I} \int_{\Omega I f} \mathbf{D}\left(\mathbf{v}_{\mathrm{p}}\right): \mathbf{D}\left(\mathbf{v}_{\mathrm{v}}\right) \mathrm{d} \omega
$$

and, according to the properties of the fields, $\left(\operatorname{div}\left(\mathbf{v}_{\mathrm{v}}\right)=0 ; \operatorname{div}\left(\sigma_{\mathrm{p}}\right)=\mathbf{0}\right)$, the right-hand member is in the form:

$$
(4 \mu) \sum_{I} \int_{\Omega_{I \mathrm{f}}} \mathbf{D}\left(\mathbf{v}_{\mathrm{p}}\right): \mathbf{D}\left(\mathbf{v}_{\mathrm{v}}\right) \mathrm{d} \omega=2 \sum_{I} \int_{\partial \Omega_{I}}\left(\sigma_{\mathrm{p}} \cdot \mathbf{n}\right) \cdot \mathbf{v}_{\mathrm{v}} \mathrm{d} s .
$$

\subsection{Bounds of permeability}

The inequality (22) is applied hereafter to media $\mathrm{PM}_{\mathrm{v}}$ and $\mathrm{PM}_{\mathrm{p}}$ related to $K^{*}$, using fields defined for a unit macroscopic pressure gradient $\operatorname{grad}(P)=\mathbf{e}_{z}$.

- the kinematically continuous field is given in each inclusion of $\mathrm{PM}_{\mathrm{v}}$ by the fields obtained using the SCM with the kinematic approach $\left(K^{*}=K_{\mathrm{v}}\right)$ :

$$
\mathbf{v}_{\mathrm{v}}=\varpi_{\mathrm{v}}^{*} \quad \text { on } \Omega_{I}, \quad \varpi_{\mathrm{v}}^{*}=\mathbf{V}=-\left(K^{*} / \mu\right) \mathbf{e}_{z} \text { on } \partial \Omega_{I},
$$


- the statically continuous field is given in each inclusion of $\mathrm{PM}_{\mathrm{p}}$ by the fields obtained using the SCM with the static approach $\left(K^{*}=K_{\mathrm{p}}\right)$ :

$$
\mathbf{v}_{\mathrm{p}}=\varpi_{\mathrm{p}}^{*} \quad \text { on } \Omega_{I}, \quad \sigma_{\mathrm{p}}^{*} \cdot \mathbf{n}=-P . \mathbf{n} \quad \text { on } \partial \Omega_{I},
$$

- the (unknown) exact field in media $\mathrm{PM}_{\mathrm{v}}: \mathbf{v}^{\mathrm{v}} \quad\left(\int_{\Omega_{I}} \mathbf{v}^{\mathrm{v}} \mathrm{d} \omega=\Omega_{I} \mathbf{V}^{\mathrm{v}}=-\Omega_{I}\left(K^{\mathrm{v}} / \mu\right) \mathbf{e}_{z}\right)$

$$
\text { and in media } \mathrm{PM}_{\mathrm{p}}: \mathbf{v}^{\mathrm{p}} \quad\left(\int_{\Omega_{I}} \mathbf{v}^{\mathrm{p}} \mathrm{d} \omega=\Omega_{I} \mathbf{V}^{\mathrm{p}}=-\Omega_{I}\left(K^{\mathrm{p}} / \mu\right) \mathbf{e}_{z}\right) \text {. }
$$

\subsubsection{Lower bound for media $\mathrm{PM}_{\mathrm{v}}$}

First consider inequality $2 E_{\mathrm{c}}\left(\mathbf{v}^{\mathrm{v}}, \varpi_{\mathrm{v}}^{*}\right)>0$ applied to medium $\mathrm{PM}_{\mathrm{v}}$. For each inclusion the volume integrals in (23) are now written:

$$
2 \mu \int_{\Omega_{\mathrm{f}}} \mathbf{D}\left(\mathbf{v}^{\mathrm{v}}\right): \mathbf{D}\left(\mathbf{v}^{\mathrm{v}}\right) \mathrm{d} \omega=\Omega_{I} K^{\mathrm{v}} / \mu, \quad 2 \mu \int_{\Omega_{\mathrm{f}}} \mathbf{D}\left(\varpi_{\mathrm{v}}^{*}\right): \mathbf{D}\left(\varpi_{\mathrm{v}}^{*}\right) \mathrm{d} \omega=\Omega_{I} K^{*} / \mu
$$

and the surface integral (24) becomes:

$$
\begin{aligned}
\int_{\partial \Omega_{I}}\left(\sigma^{\mathrm{v}} \cdot \mathbf{n}\right) \cdot \varpi_{\mathrm{v}}^{*} \mathrm{~d} s & =\int_{\partial \Omega_{I}}\left(\sigma^{\mathrm{v}} \cdot \mathbf{n}\right) \cdot \mathbf{V} \mathrm{d} s=\mathbf{V} \cdot \int_{\partial \Omega_{I}} \sigma^{\mathrm{v}} \cdot \mathbf{n} \mathrm{d} s \\
& =-\mathbf{V} \cdot \int_{\partial \Omega_{I}} P \cdot \mathbf{n} \mathrm{d} s=-\mathbf{V} \cdot \int_{\Omega_{I}} \operatorname{grad}(P) \mathrm{d} \omega=-\Omega_{I} \operatorname{grad}(P) \cdot \mathbf{V}=\Omega_{I} K^{*} / \mu .
\end{aligned}
$$

By summing up the volume and surface terms corresponding to each inclusion and dividing the value thus obtained by the total volume, the inequality $2 E_{\mathrm{c}}\left(\mathbf{v}^{\mathrm{v}}, \varpi_{\mathrm{v}}^{*}\right)>0$ becomes

$$
K^{\mathrm{v}}+K^{*}>2 K^{*} \quad \text { i.e.: } \quad K^{\mathrm{v}}>K^{*}=K_{\mathrm{v}} \text {. }
$$

\subsubsection{Upper bound for media $\mathrm{PM}_{\mathrm{p}}$}

Now consider inequality $2 E_{\mathrm{c}}\left(\varpi_{\mathrm{p}}^{*}, \mathbf{v}^{\mathrm{p}}\right)>0$ applied to medium $\mathrm{PM}_{\mathrm{p}}$. The volume integrals in (23) are respectively:

$$
2 \mu \int_{\Omega_{\mathrm{f}}} \mathbf{D}\left(\varpi_{\mathrm{p}}^{*}\right): \mathbf{D}\left(\varpi_{\mathrm{p}}^{*}\right) \mathrm{d} \omega=\Omega_{I} K^{*} / \mu, \quad 2 \mu \int_{\Omega_{\mathrm{f}}} \mathbf{D}\left(\mathbf{v}^{\mathrm{p}}\right): \mathbf{D}\left(\mathbf{v}^{\mathrm{p}}\right) \mathrm{d} \omega=\Omega_{I} K^{\mathrm{p}} / \mu
$$

and the surface integrals (24) become:

$$
\begin{aligned}
\int_{\partial \Omega_{I}}\left(\sigma_{\mathrm{p}}^{*} \cdot \mathbf{n}\right) \cdot \mathbf{v}^{\mathrm{p}} \mathrm{d} s & =\int_{\partial \Omega_{I}}(-P \cdot \mathbf{n}) \cdot \mathbf{v}^{\mathrm{p}} \mathrm{d} s \\
& =\int_{\Omega_{I}}-\operatorname{grad}(P) \cdot \mathbf{v}^{\mathrm{p}} \mathrm{d} \omega=-\operatorname{grad}(P) \cdot \int_{\Omega_{I}} \mathbf{v}^{\mathrm{p}} \mathrm{d} \omega=-\Omega_{I} \operatorname{grad}(P) \cdot \mathbf{V}^{\mathrm{p}}=\Omega_{I} K^{\mathrm{p}} / \mu,
\end{aligned}
$$

i.e. inequality $2 E_{\mathrm{c}}\left(\mathbf{v}^{\mathrm{v}}, \varpi_{\mathrm{v}}\right)>0$ gives:

$$
K^{*}+K^{\mathrm{p}}>2 K^{\mathrm{p}}, \quad \text { i.e.: } \quad K_{\mathrm{p}}=K^{*}>K^{\mathrm{p}} .
$$


In conclusion the kinematic assumption underestimates the actual permeability $K^{\mathrm{v}}$ of any medium $\mathrm{PM}_{\mathrm{v}}$, and the static assumption overestimates the actual permeability $K^{\mathrm{p}}$ of any medium $\mathrm{PM}_{\mathrm{p}}$.

$$
K^{\mathrm{v}}>K_{\mathrm{v}}, \quad K_{\mathrm{p}}>K^{\mathrm{p}} .
$$

\subsection{Bounds for a large class of porous media}

The method developed above is extended to any porous media PM, the structure of which being an arrangement of spheres filling the whole space. Each sphere contains a solid concentric sphere, as already described above. Unlike for media $\mathrm{PM}_{\mathrm{v}}$ and $\mathrm{PM}_{\mathrm{p}}$ no additional conditions are imposed to the inclusions constituting the medium PM. We assume that the medium presents an elementary representative volume ERV constituted by inclusions and that its permeability is $K$. The volume $\Omega_{\mathrm{ERV}}$ is given by:

$$
\Omega_{\mathrm{ERV}}=\left\{\sum_{I} R_{I}^{3}\right\}(4 \pi / 3)
$$

To establish theoretical bounds for permeability, we built a kinematically and a statically continuous field adapted to medium PM submitted to a unit macroscopic pressure $\operatorname{gradient} \operatorname{grad}(P)=\mathbf{e}_{z}$. For both fields the Navier-Stokes equation, as well as the incompressibility and the adherence condition, are fulfilled in the fluid of each inclusion.

\subsubsection{Kinematically continuous field $\mathbf{v}_{\mathrm{v}}$}

In order to ensure the kinematic continuity of $\mathbf{v}_{\mathrm{v}}$, we must have at the boundary of each inclusion: $\mathbf{v}_{\mathrm{v}}=\mathbf{V}=-(K / \mu) \mathbf{e}_{z}$. In inclusions $I$, we use kinematic fields $\varpi_{\mathrm{v}}$ determined in section 3.2. To fit the imposed kinematic condition, in each inclusion $\mathrm{I}(\rho, R), \mathbf{v}_{\mathrm{v}}$ takes the value $\mathbf{v}_{\mathrm{v} I}=\left(K / K_{\mathrm{v} I}\right) \varpi_{\mathrm{v}}$. Then $\mathbf{v}_{\mathrm{v}}$ is completely defined in the medium. The related dissipated power for the representative volume ERV, $E_{\mathrm{V}}$, can be thus calculated and takes the form, where $H_{\mathrm{v}}$ has the dimension of an intrinsic permeability:

$$
\begin{aligned}
E_{\mathrm{v}}=\Omega_{\mathrm{ERV}} H_{\mathrm{v}} / \mu & =\sum_{I}\left\{2 \mu \int_{\Omega_{\mathrm{f} I}} \mathbf{D}\left(\mathbf{v}_{\mathrm{v} I}\right): \mathbf{D}\left(\mathbf{v}_{\mathrm{v} I}\right) \mathrm{d} \omega\right\} \\
& =\sum_{I}\left\{2 \mu\left(K / K_{\mathrm{v} I}\right)^{2} \int_{\Omega_{\mathrm{f} I}} \mathbf{D}\left(\varpi_{\mathrm{v}}\right): \mathbf{D}\left(\varpi_{\mathrm{v}}\right) \mathrm{d} \omega\right\} \\
& =\left(K^{2} / \mu\right)\left\{\sum_{I} \Omega_{I} / K_{\mathrm{v} I}\right\}=\left(K^{2} / \mu\right)\left\{\sum_{I} R_{I} / \Psi_{\mathrm{v}}\left(\rho_{I}\right)\right\}(4 \pi / 3) .
\end{aligned}
$$

\subsubsection{Statically continuous field $\mathbf{v}_{\mathrm{p}}$}

Concerning $\mathbf{v}_{\mathrm{p}}$, the static continuity is obtained by imposing at the boundary of inclusions: $\sigma_{\mathrm{p}} . \mathbf{n}=P . \mathbf{n}$. In each inclusion $I(\rho, R), \mathbf{v}_{\mathrm{p}}$ is directly given by $\mathbf{v}_{\mathrm{p} I}=\varpi_{\mathrm{p}}$ which fits the imposed static condition. The related dissipated power for the representative volume ERV, $E_{\mathrm{p}}$, takes the form, where $H_{\mathrm{p}}$ has the dimension of an intrinsic permeability:

$$
\begin{aligned}
E_{\mathrm{p}}=\Omega_{\mathrm{ERV}} H_{\mathrm{p}} / \mu & =\sum_{I}\left\{2 \mu \int_{\Omega_{\mathrm{f} I}} \mathbf{D}\left(\mathbf{v}_{\mathrm{p} I}\right): \mathbf{D}\left(\mathbf{v}_{\mathrm{p} I}\right) \mathrm{d} \omega\right\} \\
& =\left\{\sum_{I} \Omega_{I} K_{\mathrm{p} I}\right\} / \mu=\left\{\sum_{I} R_{I}^{5} \Psi_{\mathrm{p}}\left(\rho_{I}\right)\right\}(4 \pi / 3) / \mu .
\end{aligned}
$$




\subsubsection{Upper bound}

The upper bound is deduced from inequality $2 E_{\mathrm{c}}\left(\mathbf{v}_{\mathrm{p}}, \mathbf{v}\right)>0$ applied to the representative volume ERV, $\mathbf{v}$ being the exact solution in the ERV of medium PM submitted to the unit macroscopic pressure gradient $\operatorname{grad}(P)=\mathbf{e}_{z}$. The dissipated powers due to fields $\mathbf{v}_{\mathrm{p}}$ and $\mathbf{v}$ are respectively $\Omega_{\mathrm{ERV}} H_{\mathrm{p}} / \mu$ and $\Omega_{\mathrm{ERV}} K / \mu$. The coupled power at each inclusion boundary is:

$$
\begin{aligned}
\int_{\partial \Omega_{I}}\left(\sigma_{\mathrm{p}} \cdot \mathbf{n}\right) \cdot \mathbf{v} \mathrm{d} s & =\int_{\partial \Omega_{I}}(-P \cdot \mathbf{n}) \cdot \mathbf{v} \mathrm{d} s \\
& =\int_{\Omega_{I}}-\operatorname{grad}(P) \cdot \mathbf{v} \mathrm{d} \omega=-\operatorname{grad}(P) \cdot \int_{\Omega_{I}} \mathbf{v} \mathrm{d} \omega=-\Omega_{I} \operatorname{grad}(P) \cdot \mathbf{V}=\Omega_{I} K / \mu .
\end{aligned}
$$

By summing up all the inclusions of ERV we get: $\Omega_{\mathrm{ERV}} K / \mu$.

Thus the inequality becomes:

$$
H_{\mathrm{p}}+K>2 K, \quad \text { i.e.: } H_{\mathrm{p}}>K \text {, }
$$

i.e.

$$
K<\left\{\sum_{I} R_{I}^{5} \Psi_{\mathrm{p}}\left(\rho_{I}\right)\right\} /\left\{\sum_{I} R_{I}^{3}\right\}
$$

\subsubsection{Lower bound}

We express now that $2 E_{\mathrm{c}}\left(\mathbf{v}, \mathbf{v}_{\mathrm{v}}\right)>0$ for the representative volume ERV. The dissipated powers due to fields $\mathbf{v}_{\mathrm{v}}$ and $\mathbf{v}$ are respectively $\Omega_{\mathrm{ERV}} H_{\mathrm{v}} / \mu$ and $\Omega_{\mathrm{ERV}} K / \mu$. The coupled power at each inclusion boundary is:

$$
\begin{aligned}
\int_{\partial \Omega_{I}}(\sigma \cdot \mathbf{n}) \cdot \mathbf{v}_{\mathrm{V}} \mathrm{d} s & =\int_{\partial \Omega_{I}}(\sigma \cdot \mathbf{n}) \cdot \mathbf{V} \mathrm{d} s=\mathbf{V} \cdot \int_{\partial \Omega_{I}} \sigma \cdot \mathbf{n} \mathrm{d} s \\
& =-\mathbf{V} \cdot \int_{\partial \Omega_{I}} P \cdot \mathbf{n} \mathrm{d} s=-\mathbf{V} \cdot \int_{I} \operatorname{grad}(P) \mathrm{d} \omega=-\Omega_{I} \operatorname{grad}(P) \cdot \mathbf{V}=\Omega_{I} K / \mu .
\end{aligned}
$$

After the summation of all the inclusions of ERV the inequality gives:

$$
K+H_{\mathrm{v}}>2 K, \quad \text { i.e.: } H_{\mathrm{v}}>K \text {, }
$$

i.e.

$$
K>\left\{\sum_{I} R_{I}^{3}\right\} /\left\{\sum_{I} R_{I} / \Psi_{\mathrm{v}}\left(\rho_{I}\right)\right\}
$$

In conclusion, the method provides a way to bound the permeability of a given porous media PM with a given known structure. The kinematically continuous field gives an underestimation, whereas the statically continuous field gives an overestimation of the actual permeability of the medium.

$$
H_{\mathrm{v}}=\left\{\sum_{I} R_{I}^{3}\right\} /\left\{\sum_{I} R_{I} / \Psi_{\mathrm{v}}\left(\rho_{I}\right)\right\}<K<\left\{\sum_{I} R_{I}^{5} \Psi_{\mathrm{p}}\left(\rho_{I}\right)\right\} /\left\{\sum_{I} R_{I}^{3}\right\}=H_{\mathrm{p}}
$$

Inversely to the lower/upper bound (25) valid for any media $\mathrm{PM}_{\mathrm{v}}$ and $\mathrm{PM}_{\mathrm{p}}$, related to $K^{*}$, in the bounds (26), $H_{\mathrm{v}}$ and $H_{\mathrm{p}}$ can be calculated only if the porous structure is known, i.e., the grain size distribution (granulometry) and the fluid shell size distribution. This is an additional demonstration of the complexity of the relationship which links the permeability and the microstructure, even in simple configurations. 


\subsubsection{Application to various classes of porous media}

Let us apply inequality (26) to porous media $\mathrm{PM}_{\mathrm{v}}$ (of actual permeability $K^{\mathrm{v}}$ ). In this case (by construction) we get for all the inclusions: $\Psi_{\mathrm{v}}\left(\rho_{I}\right) R_{I}^{2}=K_{\mathrm{v}}$. Thus, as already established in section 4.2.: $H_{\mathrm{v}}=K_{\mathrm{v}}$. Now, dividing (26) by $K_{\mathrm{v}}$ yields:

$$
1<K^{\mathrm{v}} / K_{\mathrm{v}}<\left\{\sum_{I} R_{I}^{5} \Psi_{\mathrm{p}}\left(\rho_{I}\right)\right\} /\left\{\sum_{I} R_{I}^{3}\right\} K_{\mathrm{v}}=\left\{\sum_{I} R_{I}^{3}\left[\Psi_{\mathrm{p}}\left(\rho_{I}\right) / \Psi_{\mathrm{v}}\left(\rho_{I}\right)\right]\right\} /\left\{\sum_{I} R_{I}^{3}\right\} .
$$

But, we have seen in section 3.4 that: $1<\Psi_{\mathrm{p}}\left(\rho_{I}\right) / \Psi_{\mathrm{v}}\left(\rho_{I}\right)<4$, which implies:

$$
1<K^{\mathrm{v}} / K_{\mathrm{v}}<4 \text {. }
$$

The same reasoning applies to medium $\mathrm{PM}_{\mathrm{p}}$ (of actual permeability $K^{\mathrm{p}}$ ) for which $\Psi_{\mathrm{p}}\left(\rho_{I}\right) R_{I}^{2}=K_{\mathrm{p}}$, first gives, $H_{\mathrm{p}}=K_{\mathrm{p}}$, and then, dividing (26) by $K_{\mathrm{p}}$ :

$$
1>K^{\mathrm{p}} / K_{\mathrm{p}}>\left\{\sum_{I} R_{I}^{3}\right\} /\left\{\sum_{I} R_{I} / \Psi_{\mathrm{v}}\left(\rho_{I}\right)\right\} K_{\mathrm{p}}=\left\{\sum_{I} R_{I}^{3}\right\} /\left\{\sum_{I} R_{I}^{3}\left[\Psi_{\mathrm{p}}\left(\rho_{I}\right) / \Psi_{\mathrm{v}}\left(\rho_{I}\right)\right]\right\}
$$

and, for the same reason:

$$
1 / 4<K^{\mathrm{p}} / K_{\mathrm{p}}<1 .
$$

Inequalities (27) and (28) are of great interest since they are valid for any medium $\mathrm{PM}_{\mathrm{v}}$ and $\mathrm{PM}_{\mathrm{p}}$ whatever the spatial distribution of the inclusions, and the inclusion size distribution. Considering these elements, it seems that despite the factor 4 between the bounds in equations (27)-(28), the range extent is rather narrow.

Nevertheless the bounds can be improved when considering a medium with dilute concentration of solid particles $\left(\rho_{I} \ll 1\right)$ so that the use of limit values for $\Psi_{\mathrm{p}}$ and $\Psi_{\mathrm{v}}$ is valid and $\Psi_{\mathrm{p}}\left(\rho_{I}\right) / \Psi_{\mathrm{v}}\left(\rho_{I}\right)=1+(3 / 4) \rho_{I}$. Then we get:

$$
\begin{aligned}
& 1<K^{\mathrm{v}} / K_{\mathrm{v}}<1+(3 / 4)\left\{\sum_{I} R_{I}^{3} \rho_{I}\right\} /\left\{\sum_{I} R_{I}^{3}\right\}, \\
& 1-(3 / 4)\left\{\sum_{I} R_{I}^{3} \rho_{I}\right\} /\left\{\sum_{I} R_{I}^{3}\right\}<K^{\mathrm{p}} / K_{\mathrm{p}}<1 .
\end{aligned}
$$

Therefore in the case of a dilute array of fixed particles, $K_{\mathrm{v}}$ and $K_{\mathrm{p}}$ are, respectively, good approximations of the actual permeability of media $\mathrm{PM}_{\mathrm{v}}$ and $\mathrm{PM}_{\mathrm{p}}$. Note that for very weak solid concentrations, this mathematical inequality is no more valid physically since the Brinkman's law has to be used instead of the Darcy law (Levy, 1983).

As a last example let consider a medium, the ERV of which being constituted by one fixed solid sphere surrounded by the fluid. Such medium corresponds to a medium PM constituted by a single inclusion $I_{0}\left(\rho_{0}, R_{0}\right)$, and by other fully fluid inclusions $I\left(0, R_{I}\right)$ to complete the ERV. Then we have (where $c$ is the solid concentration in the ERV):

$$
H_{\mathrm{v}}=3 \Omega_{\mathrm{ERV}} \Psi_{\mathrm{v}}\left(\rho_{0}\right) / 4 \pi R_{0}=\left[\rho_{0} \Psi_{\mathrm{v}}\left(\rho_{0}\right)\right]\left(R_{0} \rho_{0}\right)^{2} / c<K<H_{\mathrm{p}}=\infty .
$$

The fact that the second bound is infinite shows that the approximated fields are much less pertinent when the microstructure deviates from those of media $\mathrm{PM}_{\mathrm{v}}$ or $\mathrm{PM}_{\mathrm{p}}$. 


\subsection{Fibrous porous media}

The previous analysis can be applied to fibrous materials. Considering cylindrical fluid-solid inclusions (figure 6), and a flow parallel or perpendicular to the cylinder, conjectured values of transverse and longitudinal permeability are established, based either on the kinematic or static assumption. Calculation steps are given in the Appendix, and analytical values of $K_{\mathrm{Tv}}, K_{\mathrm{Tp}}, K_{\mathrm{Lv}}$ and $K_{\mathrm{Lp}}$ are, respectively, given by A2, A3, A5 and A6, which introduce functions $\Phi_{\mathrm{Tv}}, \Phi_{\mathrm{Tp}}, \Phi_{\mathrm{Lv}}, \Phi_{\mathrm{Lp}}$, plotted in figures 7 and 8 , similar to functions $\Psi_{\mathrm{v}}$ and $\Psi_{\mathrm{p}}$.

As for spherical inclusions, fibrous media FM constituted by an arrangement of parallel cylinders of radius $R_{n}$ filling all the space can be built, each of them containing a solid coaxial cylinder. Among these media FM, the media $\mathrm{FM}_{\mathrm{Tv}}, \mathrm{FM}_{\mathrm{Tp}}$, related to $K_{\mathrm{T}}^{*}$, and media $\mathrm{FM}_{\mathrm{Lv}}, \mathrm{FM}_{\mathrm{Lp}}$, related to $K_{\mathrm{L}}^{*}$, are obtained when the radius of the solid coaxial cylinders are such that: $\Phi_{\mathrm{Tv}}\left(\rho_{n}\right) R_{n}^{2}=K_{\mathrm{T}}^{*}\left(\right.$ medium $\left.\mathrm{FM}_{\mathrm{Tv}}\right), \Phi_{\mathrm{Tp}}\left(\rho_{n}\right) R_{n}^{2}=K_{\mathrm{T}}^{*}\left(\right.$ medium FM $\left.\mathrm{FM}_{\mathrm{Tp}}\right)$, etc.

For any fibrous medium FM, the following bounds are obtained for the transverse and longitudinal intrinsic permeabilities:

$$
\begin{aligned}
& H_{\mathrm{Tv}}=\left\{\sum_{I} R_{I}^{2}\right\} /\left\{\sum_{I} R_{I} / \Phi_{\mathrm{Tv}}\left(\rho_{I}\right)\right\}<K_{\mathrm{T}}<\left\{\sum_{I} R_{I}^{3} \Phi_{\mathrm{Tp}}\left(\rho_{I}\right)\right\} /\left\{\sum_{I} R_{I}^{2}\right\}=H_{\mathrm{Tp}}, \\
& H_{\mathrm{Lv}}=\left\{\sum_{I} R_{I}^{2}\right\} /\left\{\sum_{I} 1 / \Phi_{\mathrm{Lv}}\left(\rho_{I}\right)\right\}<K_{\mathrm{L}}<\left\{\sum_{I} R_{I}^{4} \Phi_{\mathrm{Lp}}\left(\rho_{I}\right)\right\} /\left\{\sum_{I} R_{I}^{2}\right\}=H_{\mathrm{Lp}} .
\end{aligned}
$$

Note: In the case of a transverse flow, the pressure gradient is perpendicular to the cylinder axis (direction $\mathbf{e}_{z}$ ), and the problem is fully determined in a $(r, \theta)$ plane of zero thickness in direction $\mathbf{e}_{z}$ (figure 6 ). Then the intrinsic permeability is related to a flow per length perpendicular to the pressure gradient in the $(r, \theta)$ plane, instead of an usual flow per area. That is the reason why $K_{\mathrm{T}}$ have the dimension of a length (instead of square length). In order to recover the usual dimension of intrinsic permeability $\left(\mathrm{m}^{2}\right)$, we have to consider a unit length of material in the cylinder direction $\mathbf{e}_{z}$. In the SI, the actual value of the intrinsic permeability of the medium (expressed in $\left.\mathrm{m}^{2}\right)$ is $K_{\mathrm{T}}$. $(1 \mathrm{~m})$. It should be noted that this exact value differs from the expressions proposed in a number of papers where the length unit, the meter, is arbitrarily replaced by the radius of fibres.

Taking into account the properties of functions $\Phi$, the application of (29) and (30) to media $\mathrm{FM}_{\mathrm{Tv}}, \mathrm{FM}_{\mathrm{Tp}}$, and $\mathrm{FM}_{\mathrm{Lv}}, \mathrm{FM}_{\mathrm{Lp}}$, gives the bounds:
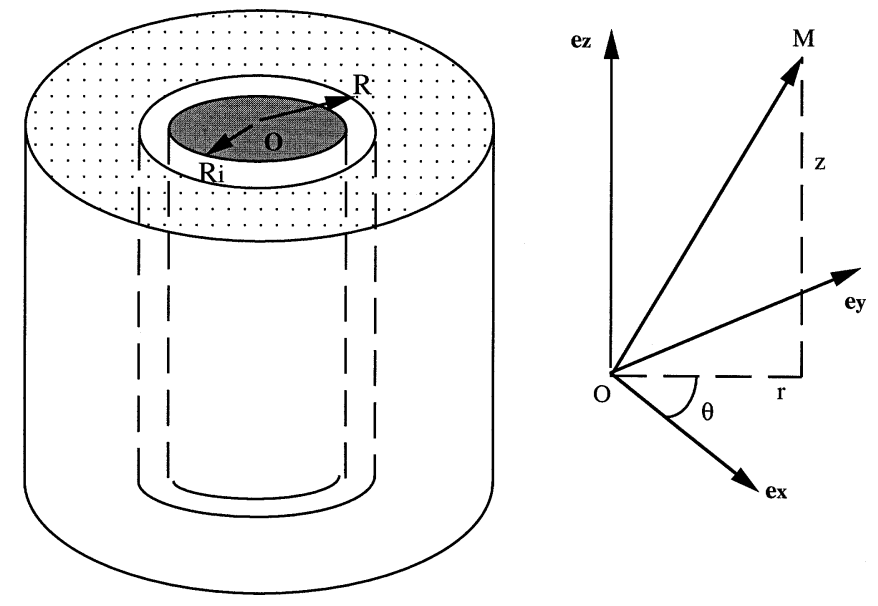

Figure 6. Generic fluid-solid cylindrical inclusion $I$ of volume $\Omega_{I}$ and boundary $\partial \Omega_{I}$ and the cylindrical system of co-ordinates. Solid cylinder: radius $R_{i}$, volume $\Omega_{\mathrm{s}}$. Coaxial cylindrical shell filled by the viscous Newtonian fluid: external radius $R$ and volume $\Omega_{\mathrm{f}}=\Omega_{I}-\Omega_{\mathrm{s}}$. 


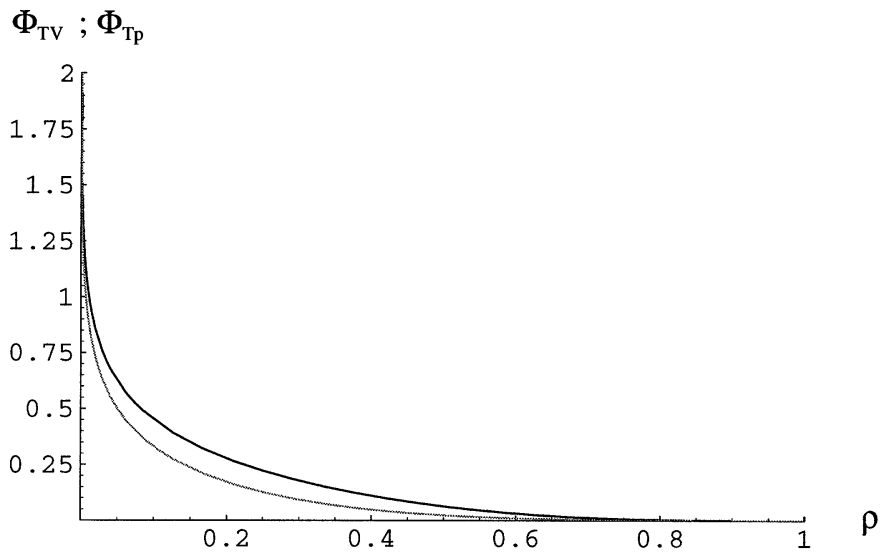

\section{$\log \left(\Phi_{\mathrm{TV}}\right) ; \log \left(\Phi_{\mathrm{Tp}}\right)$}
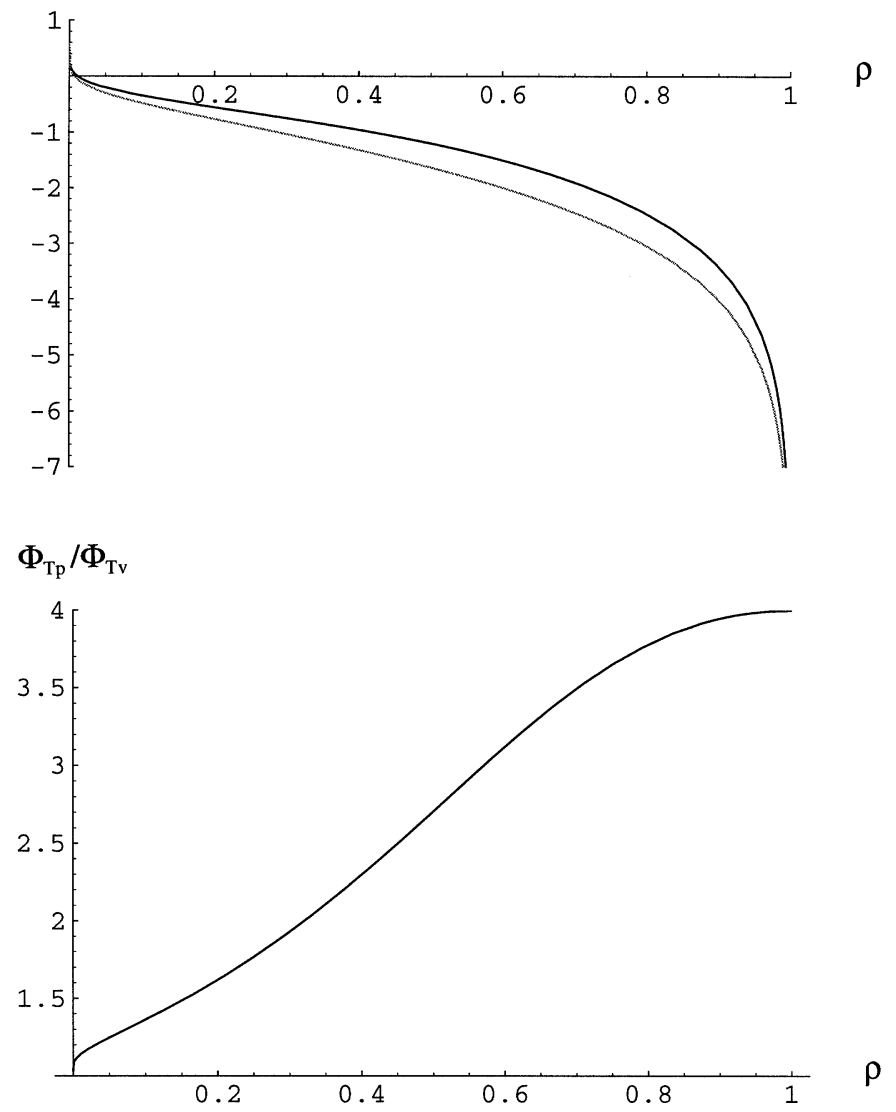

Figure 7. Cylindrical inclusions. Flow perpendicular to the cylinder axis. Functions $\Phi_{\mathrm{Tp}}$ (straight line) and $\Phi_{\mathrm{Tv}}\left(\mathrm{dashed}\right.$ line). Top: $\Phi_{\mathrm{Tp}}$ and $\Phi_{\mathrm{Tv}}$ versus $\rho$. Middle: $\log \left(\Phi_{\mathrm{Tp}}\right)$ and $\log \left(\Phi_{\mathrm{Tv}}\right)$ versus $\rho\left(\log\right.$ decimal). Bottom: Ratio $\Phi_{\mathrm{Tp}} / \Phi_{\mathrm{Tv}}$ versus $\rho$. 


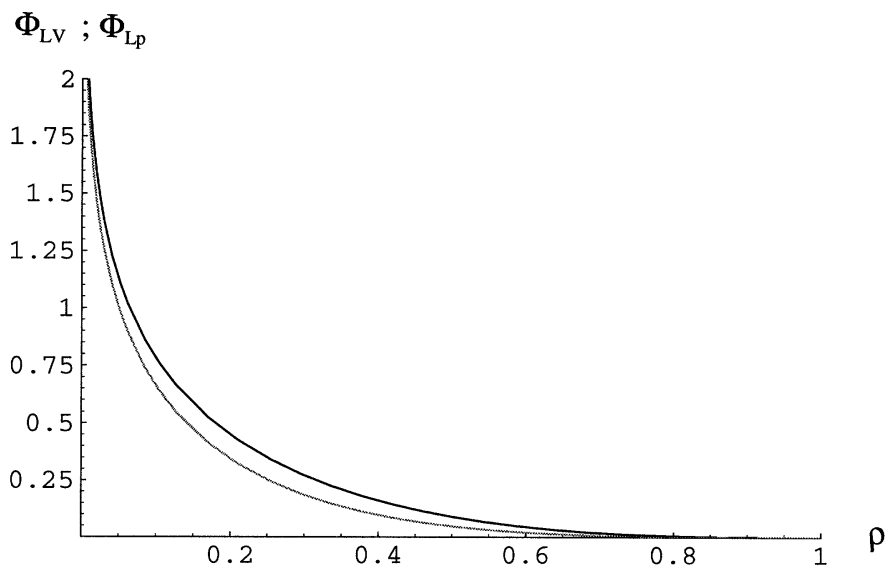

$\log \left(\Phi_{\mathrm{LV}}\right) ; \log \left(\Phi_{\mathrm{Lp}}\right)$
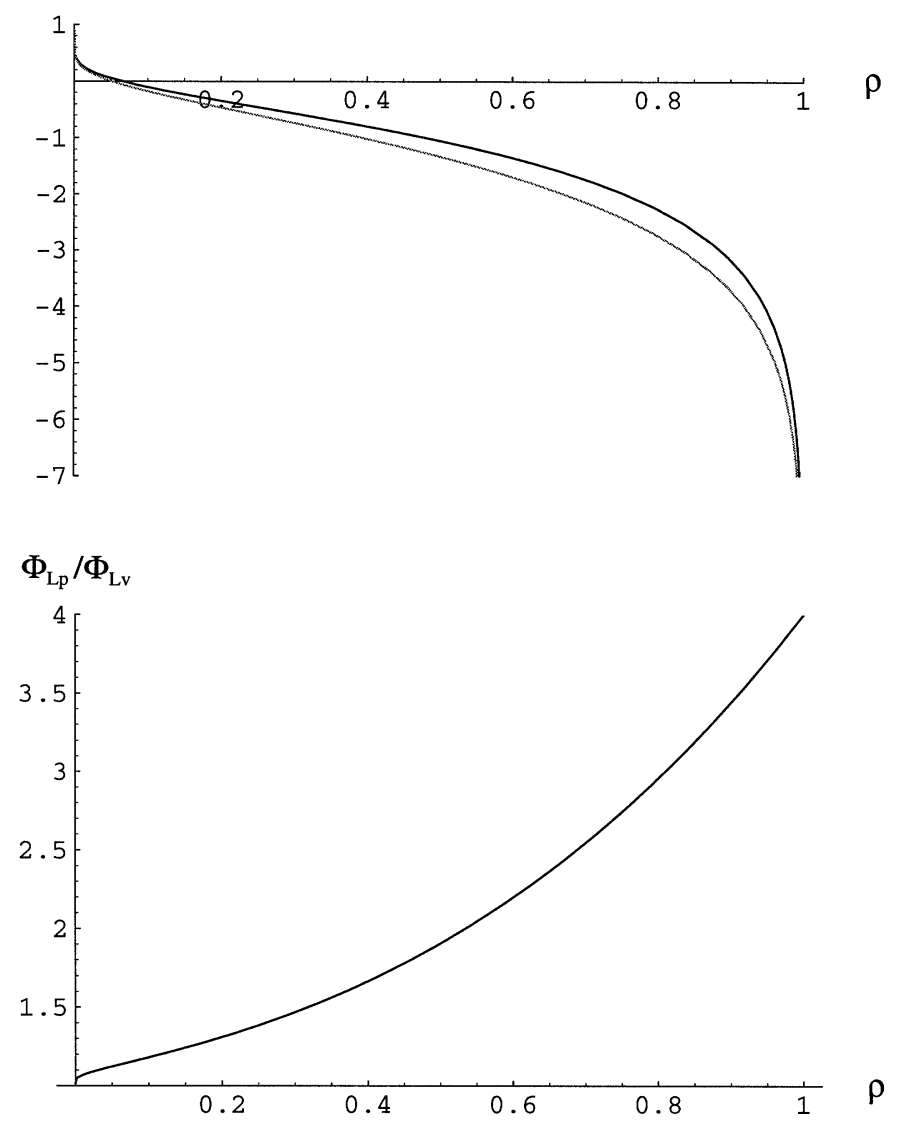

Figure 8. Cylindrical inclusions. Flow parallel to the cylinder axis. Functions $\Phi_{\mathrm{Lp}}$ (straight line) and $\Phi_{\mathrm{Lv}}$ (dashed line). Top: $\Phi_{\mathrm{Lp}}$ and $\Phi_{\mathrm{Lv}}$ versus $\rho$. Middle: $\log \left(\Phi_{\mathrm{Lp}}\right)$ and $\log \left(\Phi_{\mathrm{Lv}}\right)$ versus $\rho$ (Log decimal). Bottom: Ratio $\Phi_{\mathrm{Lp}} / \Phi_{\mathrm{Lv}}$ versus $\rho$. 


$$
\begin{array}{ll}
1<K_{\mathrm{T}}^{\mathrm{v}} / K_{\mathrm{Tv}}<4, & 1 / 4<K_{\mathrm{L}}^{\mathrm{p}} / K_{\mathrm{Lp}}<1, \\
1<K_{\mathrm{L}}^{\mathrm{v}} / K_{\mathrm{Lv}}<4, & 1 / 4<K_{\mathrm{L}}^{\mathrm{p}} / K_{\mathrm{Lp}}<1 .
\end{array}
$$

Finally, for weak solid density of fibrous material, which is very often encountered in noise absorbing medium, we have:

$$
\begin{aligned}
& 1<K_{\mathrm{T}}^{\mathrm{v}} / K_{\mathrm{Tv}}<1-(1 / 2)\left\{\sum_{I} R_{I}^{2} / \operatorname{Ln}\left(\rho_{I}\right)\right\} /\left\{\sum_{I} R_{I}^{2}\right\}, \\
& 1+(1 / 2)\left\{\sum_{I} R_{I}^{2} / \operatorname{Ln}\left(\rho_{I}\right)\right\} /\left\{\sum_{I} R_{I}^{2}\right\}<K_{\mathrm{T}}^{\mathrm{p}} / K_{\mathrm{Tp}}<1, \\
& 1<K_{\mathrm{L}}^{\mathrm{v}} / K_{\mathrm{Lv}}<1-(1 / 4)\left\{\sum_{I} R_{I}^{2} / \operatorname{Ln}\left(\rho_{I}\right)\right\} /\left\{\sum_{I} R_{I}^{2}\right\}, \\
& 1+(1 / 4)\left\{\sum_{I} R_{I}^{2} / \operatorname{Ln}\left(\rho_{I}\right)\right\} /\left\{\sum_{I} R_{I}^{2}\right\}<K_{\mathrm{L}}^{\mathrm{p}} / K_{\mathrm{Lp}}<1 .
\end{aligned}
$$

\subsection{Periodic spatial distribution}

The bounds established in this section are independent of the spatial distribution of the inclusions. Therefore they are valid for periodic media and they can be applied within the HPM framework. Since the sphere (or cylinder) size distribution is filling the whole space, this enables us to consider complex microstructures with a finite maximum value for the inclusion size. Such a medium presents a finite ERV, the structure of which can be built using auto similar structure such as those studied by Gilbert (1987).

Moreover the analytical solution established for local fields can be used as first approximated solution in numerical simulations, and provides a reference field for testing numerical codes.

\section{Conclusion}

Though the microscopic and macroscopic governing equations are rather different, SCM can still be applied. However in this case the nature of the macroscopic law cannot be derived from SCM. The rigorous physical analysis performed with the HPM therefore plays a determining role. In the studied problem, it is clear that other results would have been obtained if, instead of the Darcy law, the Brinkman's law, which is actually pertinent for very dilute array of particles (Levy, 1983) was assumed, or if a viscous behaviour, which is the required approach for fluids of very high viscosity (Boutin and Auriault, 1990) was assumed. Note that the results obtained are expressed in terms of intrinsic permeability, which only depends on the pores geometry, and they are also applicable to linear viscoelastic saturating fluids.

The conjectured value given by SCM is generally taken as a good approximation of the macroscopic properties of a medium presenting the same fluid-solid concentration as the generic inclusion (see for example Berdichevsky and Cai (1993); Tarnow (1996)). This study shows that for the permeability assessment the situation is actually more complex for two reasons:

- first, the size of inclusions explicitly intervenes in the result,

- second, two values can be conjectured.

Reasoning based on the comparison of the dissipated power related on the one hand to the exact solution, and on the second hand to kinematically and statically continuous fields proves that SCM conjectured values 
constitute the bounds of permeability. The analysis of approximated fields at the micro-scale allows the identification of two classes of porous media for which bounds lie in a narrow range (1 to 4). The results of SCM also enable the establishment of more open bounds valid for a wide class of porous media with a clear identification of their microstructures (grain and fluid size distribution).

Finally, and perhaps beyond the permeability-related results themselves, this study demonstrated that, from a methodological point of view, the combined use of both HPM and SCM provides an interesting approach for investigating the properties of heterogeneous media in various physical domains.

\section{Appendix A. Cylindrical fluid-solid inclusions}

We consider here the case of fibrous porous media. The generic inclusion is now constituted by a solid cylinder of radius $R_{i}$ surrounded by a fluid shell of external radius $R$. This inclusion is embedded in a Darcy medium. Because of the cylindrical symmetry we use the cylindrical co-ordinates $(r, \theta, z)$ oriented as described in figure 6. As in section 3, the governing equations in the Darcy domain $(r>R)$ are given by (8), and in the fluid shell $\left(R_{i}<r<R\right)$ by (9).

It is not necessary to reproduce all the steps of the above demonstration because the reasoning for spherical inclusions is similar. Therefore we directly start with an unmodified field in the Darcy medium:

$$
\operatorname{grad}\left(P^{\mathrm{e}}\right)=\mathbf{e} \quad \text { and } \quad \mathbf{V}^{\mathrm{e}}=-(K / \mu) \mathbf{e} .
$$

The cylindrical symmetry requires to distinguish the following cases:

- flow perpendicular to the cylinder, i.e. when $\mathbf{e}=\mathbf{e}_{x}\left(\mathbf{e}_{r}=\mathbf{e}_{x}\right.$ for $\left.\theta=0\right)$,

- flow parallel to the cylinder, i.e. when $\mathbf{e}=\mathbf{e}_{z}$.

\section{A1. Flow perpendicular to the cylinder axis}

According to the symmetry of the problem, the field in the fluid shell $\left(R>r>R_{i}\right)$ is independent of $z$, and then is written in the form:

$$
\mathbf{v}=\left\{\begin{array}{l}
v_{r}=-(K / \mu) f_{\mathrm{T}}(r) \cos (\theta), \\
v_{\theta}=(K / \mu) g_{\mathrm{T}}(r) \sin (\theta), \quad p=K h_{\mathrm{T}}(r) \cos (\theta) . \\
v_{z}=0,
\end{array}\right.
$$

The general expressions of the functions $h_{\mathrm{T}}, f_{\mathrm{T}}, g_{\mathrm{T}}$, solutions are:

$$
\begin{aligned}
h_{\mathrm{T}}(r) & =\left[-2 b(R / r)+8 d(R / r)^{-2}\right] / r, \\
f_{\mathrm{T}}(r) & =a(R / r)^{2}+b \operatorname{Ln}(r / R)+c+d(R / r)^{-2}, \\
g_{\mathrm{T}}(r) & =-a(R / r)^{2}+b \operatorname{Ln}(r / R)+b+c+3 d(R / r)^{-2}
\end{aligned}
$$

they meets the relations: $-h_{\mathrm{T}}+f_{\mathrm{T}}^{\prime}+g_{\mathrm{T}}^{\prime}=4 b / r, f_{\mathrm{T}}^{\prime}+2\left(f_{\mathrm{T}}-g_{\mathrm{T}}\right) / r=0$.

Kinematic approach: field $\varpi_{\mathrm{Tv}}$

As for the case of a spherical inclusion, under the assumption of kinematics continuity, the conditions to be expressed for determining the four parameters $(a, b, c, d)$ and unknown $K_{\mathrm{Tv}}$ are:

- the adherence condition at the fluid-solid interface $\left(v\left(R_{i}\right)=0\right)$, 
- the flow continuity at the Darcy-fluid interface $\left(\mathbf{v}(R)=\mathbf{V}^{\mathrm{e}}\right)$,

- and finally the condition resulting from the overall equilibrium (14), which takes the following form for a flow perpendicular to the cylindrical inclusion:

$$
R=K\left\{-h_{\mathrm{T}}(R)+f_{\mathrm{T}}^{\prime}(R)+g_{\mathrm{T}}^{\prime}(R)\right\} .
$$

Then, the set to be solved is:

$$
\begin{array}{ll}
a \rho^{-2}+b \operatorname{Ln}(\rho)+c+d \rho^{2}=0, & v_{r}\left(R_{i}\right)=0, \\
-a \rho^{-2}+b(\operatorname{Ln}(\rho)+1)+c+3 d \rho^{2}=0, & v_{\theta}\left(R_{i}\right)=0, \\
a+0+c+d=1, & v_{r}(R)=V_{r}^{\mathrm{e}} \\
-a+b+c+3 d=1, & v_{\theta}(R)=V_{\theta}^{\mathrm{e}}, \\
+b=R / 4 K_{\mathrm{Tv}}, & (\mathrm{A} 1) .
\end{array}
$$

Static approach: field $\varpi_{\mathrm{Tp}}$

For the static assumption, we maintain the adherence condition, but instead of the flow continuity, we express the stress continuity, i.e. $\sigma \cdot \mathbf{n}=-P^{\mathrm{e}} \mathbf{n}$, and the system is completed by the averaged flow identity $\left(v_{r}(R)=V_{r}^{\mathrm{e}}\right)$. This leads to a set of five equations:

$$
\begin{array}{ll}
a \rho^{-2}+b \operatorname{Ln}(\rho)+c+d \rho^{2}=0, & v_{r}\left(R_{i}\right)=0, \\
-a \rho^{-2}+b(\operatorname{Ln}(\rho)+1)+c+3 d \rho^{2}=0, & v_{\theta}\left(R_{i}\right)=0, \\
a+0+0+d=0, & \sigma_{r \theta}=0, \\
+b=R / 4 K_{\mathrm{Tp}}, & \sigma_{r r}(R)=P^{\mathrm{e}}(R), \\
a+0+c+d=1, & v_{r}(R)=V_{r}^{\mathrm{e}} .
\end{array}
$$

\section{Solutions}

The resolution of the kinematic and static sets yields the following kinematic and static values respectively:

$$
\begin{gathered}
K_{\mathrm{Tv}} / R=\Phi_{\mathrm{Tv}}(\rho)=-\left[\operatorname{Ln}(\rho)+\left(1-\rho^{2}\right) /\left(1+\rho^{2}\right)\right] / 4, \\
K_{\mathrm{Tp}} / R=\Phi_{\mathrm{Tp}}(\rho)=-\left[\operatorname{Ln}(\rho)+\left(1-\rho^{4}\right) / 2\left(1+\rho^{4}\right)\right] / 4 .
\end{gathered}
$$

It can be easily demonstrated that $\Phi_{\mathrm{Tp}}>\Phi_{\mathrm{Tv}}$ and that the limit values when $\rho$ tends to 0 and to 1 are:

$$
\begin{array}{ll}
\rho \rightarrow 0 & \rho \rightarrow 1 \\
\Phi_{\mathrm{Tv}}(\rho) \rightarrow-[\operatorname{Ln}(\rho)+1] / 4, & \Phi_{\mathrm{Tv}}(\rho) \rightarrow(1-\rho)^{3} / 12, \\
\Phi_{\mathrm{Tp}}(\rho) \rightarrow-[\operatorname{Ln}(\rho)+1 / 2] / 4, & \Phi_{\mathrm{Tp}}(\rho) \rightarrow(1-\rho)^{3} / 3 .
\end{array}
$$

A2. Flow parallel to the cylinder axis

In this configuration the fields are independent of $z$ and $\theta$, and the $\theta$ component vanishes. In the fluid shell the general expressions for $\mathbf{v}$ and $p$ are: 


$$
\begin{aligned}
& v_{r}=-(K / \mu) f_{\mathrm{L}}(r), \quad p=-K h_{\mathrm{L}}(z), \\
& v_{z}=-(K / \mu) g_{\mathrm{L}}(r),
\end{aligned}
$$

where:

$$
h_{\mathrm{L}}(z)=z(a / R)^{2} f_{\mathrm{L}}(r)=d(R / r), \quad g_{\mathrm{L}}(r)=b \operatorname{Ln}(r / R)+c+(a / 4)(R / r)^{-2} .
$$

It is clear that the adherence condition imposes $d=0$, then $v_{r}$ uniformly vanishes.

Kinematic approach: field $\varpi_{\mathrm{Lv}}$

If the kinematic continuity is assumed, the conditions to be expressed for determining the three remaining parameters $(a, b, c)$ and the unknown $K_{\mathrm{Lv}}$ are:

- the adherence condition at the fluid-solid interface $\left(v_{\theta}\left(R_{i}\right)=0\right)$,

- the flow continuity at the Darcy-fluid interface $\left(\mathbf{v}(R)=\mathbf{V}^{\mathrm{e}}\right)$,

- and finally the condition resulting from the overall equilibrium (14), which takes the following form for a flow parallel to the cylindrical inclusion:

$$
R=K\left\{-\mathrm{d}\left(h_{\mathrm{L}}\right) / \mathrm{d} z+2 g_{\mathrm{L}}^{\prime}(R)\right\}
$$

Then, the set to be solved is the following:

$$
\begin{array}{ll}
(a / 4) \rho^{2}+b \operatorname{Ln}(\rho)+c=0, & v_{z}\left(R_{i}\right)=0 \\
(a / 8)\left(1-\rho^{4}\right)-b\left(1 / 2+\rho^{2}(\operatorname{Ln}(\rho)-1 / 2)\right)+c\left(1-\rho^{4}\right) / 4=1, & v_{r}(R)=V_{r}^{\mathrm{e}} \\
a / 4+0+c=1, & v_{z}(R)=V_{z}^{\mathrm{e}} \\
+b=R^{2} / 2 K_{\mathrm{Lv}}, & (\mathrm{A} 4) .
\end{array}
$$

\section{Static approach: field $\varpi_{\mathrm{Lp}}$}

Under the static assumption, we maintain the adherence condition, but instead of the flow continuity, we get the stress continuity, i.e. $\sigma \cdot \mathbf{n}=-P^{\mathrm{e}} \mathbf{n}$, and the system is completed by the averaged flow identity $\left(v_{r}(R)=V_{r}^{\mathrm{e}}\right)$. This leads to the following set:

$$
\begin{array}{ll}
(a / 4) \rho^{2}+b \operatorname{Ln}(\rho)+c=0, & v_{z}\left(R_{i}\right)=0, \\
a / 2+b=0 & \sigma_{r z}=0, \\
+b=R^{2} / 2 K_{\mathrm{Lp}}, & \sigma_{r r}(R)=P^{\mathrm{e}}(R), \\
(a / 8)\left(1-\rho^{4}\right)-b\left(1 / 2+\rho^{2}(\operatorname{Ln}(\rho)-1 / 2)\right)+c\left(1-\rho^{4}\right) / 4=1, & v_{r}(R)=V_{r}^{\mathrm{e}} .
\end{array}
$$

\section{Solutions}

The resolution of the kinematic and static sets gives the kinematic and static values:

$$
\begin{aligned}
& K_{\mathrm{Lv}} / R^{2}=\Phi_{\mathrm{Lv}}(\rho)=-\left[\operatorname{Ln}(\rho)+\left(1-\rho^{2}\right) /\left(1+\rho^{2}\right)\right] / 2, \\
& K_{\mathrm{Lp}} / R^{2}=\Phi_{\mathrm{Lp}}(\rho)=-\left[\operatorname{Ln}(\rho)+\left(1-\rho^{2}\right)\left(3-\rho^{2}\right) / 4\right] / 2 .
\end{aligned}
$$


Here again it is demonstrated that $\Phi_{\mathrm{Lp}}>\Phi_{\mathrm{Lv}}$ and that the limit values when $\rho$ tends to 0 and to 1 are:

$$
\begin{array}{ll}
\rho \rightarrow 0 & \rho \rightarrow 1 \\
\Phi_{\mathrm{Lv}}(\rho) \rightarrow-[\operatorname{Ln}(\rho)+1] / 2, & \Phi_{\mathrm{Lv}}(\rho) \rightarrow(1-\rho)^{3} / 6, \\
\Phi_{\mathrm{Lp}}(\rho) \rightarrow-[\operatorname{Ln}(\rho)+3 / 4] / 2, & \Phi_{\mathrm{Lp}}(\rho) \rightarrow 2(1-\rho)^{3} / 3 .
\end{array}
$$

Notice that we find in equations (A3), (A6) the expression given by Berdichevsky and Cai (1993), which were obtained from the static continuity assumption as in (A3), (A6).

\section{References}

Auriault J.L., Heterogeneous medium. Is an equivalent macroscopic description possible? Int. J. Engng. Sc. 29 (1991) 785-795.

Auriault J.L., Sanchez-Palencia E., Etude du comportement macroscopique d'un milieu poreux saturé déformable, J. de Mécanique 16 (1977) 575-603.

Berdichevsky A.L., Cai Zhong, Perform permeability predictions by self-consistent method and finite element simulation, Polymer Composites 14 (1993) 132-143

Biot M.A., General theory of three-dimensional consolidation, J. Applied Phys. 12 (1941) 155-164.

Boutin C., Auriault J.L., Dynamic behaviour of porous media saturated by a viscoelastic fluid. Application to bituminous concretes, Int. J. Engng. Sci. 28 (1990) 1157-1181.

Christensen R.M., Lo K.H., Solution for effective shear properties in three phase sphere and cylinder models, J. Mech. Phys. Solids 27 (1979) $315-330$.

Gilbert F., Descriptions thermo-mécaniques de milieux à plusieurs constituants et applications aux milieux poreux saturés, Thèse d'état. Université P. et M. Curie, Paris VI, 1987.

Hashin Z., Assessment of self-consistent scheme approximation: Conductivity of particulate composites, J. Comp. Mater. 2 (1968) $284-304$.

Levy T., Sanchez-Palencia E., On boundary conditions for fluid flow in porous media, Int. J. Engng. Sc. 13 (1975) 923-940.

Levy T., Fluid flow through an array of fixed particles, Int. J. Engng. Sc. 21 (1983) 11-23.

Sanchez-Palencia E., Nonhomogeneous media and vibration theory, Lecture Note in Physics 127, Springer-Verlag, Berlin, 1980.

Sangani A.S., Acrivos A., Slow flow past periodic arrays of cylinders with applications to heat transfer, Int. J. Multiphase Flow 8 (1982) $193-206$.

Tarnow V., Air flow resistivity of models of fibrous acoustic materials, J. Acoust. Soc. Am. 100 (1996) 3706-3713. 